\title{
Normes invariantes et existence de filtrations admissibles
}

\author{
Yongquan $\mathrm{Hu}$
}

\begin{abstract}
Résumé - Dans [3], est formulée une conjecture sur l'équivalence entre l'existence de normes invariantes sur certaines représentations localement algébriques de $\mathrm{GL}_{d+1}(L)$ et l'existence de certaines représentations de de Rham de $\operatorname{Gal}\left(\overline{\mathbb{Q}}_{p} / L\right)$, où $L$ est une extension finie de $\mathbb{Q}_{p}$. Dans cet article, on montre le sens "facile" de cette conjecture : l'existence de normes invariantes entraîne l'existence de filtrations admissibles.
\end{abstract}

\begin{abstract}
In [3], is formulated a conjecture on the equivalence of the existence of invariant norms on certain locally algebraic representations of $\mathrm{GL}_{d+1}(L)$ and the existence of certain de Rham representations of $\operatorname{Gal}\left(\overline{\mathbb{Q}}_{p} / L\right)$, where $L$ is a finite extension of $\mathbb{Q}_{p}$. In this paper, we prove the "easy" direction of the conjecture: the existence of invariant norms implies the existence of admissible filtrations.
\end{abstract}

\section{Table des matières}

1 Introduction $\quad 2$

2 Rappels et notations 5

2.1 Notations générales . . . . . . . . . . . . . . . . . 5

2.2 Rappels de quelques constructions . . . . . . . . . . . . . 5

2.3 Rappels sur la conjecture et compléments . . . . . . . . . . . . . 8

2.4 L'ordre des $\left(r_{i}, N_{i}, V_{i}\right) \ldots \ldots \ldots \ldots \ldots \ldots$

2.5 Module de Jacquet et un lemme d'Emerton . . . . . . . . . . . . . . 11

2.6 Théorème principal . . . . . . . . . . . . . . . . . . . . . . 12

$3 \quad$ Preuve de (iii) $\Leftrightarrow$ (iv) 13

3.1 Le foncteur $r_{Q}^{G} \circ \operatorname{Ind}_{P}^{G} \ldots \ldots \ldots \ldots \ldots \ldots \ldots \ldots$

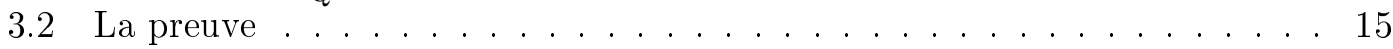

$4 \quad$ Preuve de (iii) $\Rightarrow$ (ii) 16

4.1 Construction de $\left(\varphi, N, \operatorname{Gal}\left(L^{\prime} / L\right), D\right) \ldots \ldots \ldots \ldots \ldots \ldots$

4.2 Structure de $D \ldots \ldots \ldots \ldots \ldots \ldots \ldots \ldots$

4.3 Définition de la filtration $\operatorname{sur} D \ldots \ldots \ldots \ldots \ldots \ldots \ldots \ldots \ldots$

4.4 La preuve : cas spécial . . . . . . . . . . . . . . . . 22

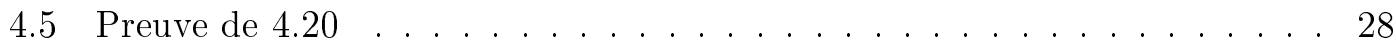

4.6 Le cas général . . . . . . . . . . . . . . . . . . . . . . 30

Mathematics Subject Classification (1991) : 05A20, 17B10, 22E50 


\section{Introduction}

L'objet de cet article est une conjecture proposée dans [3]. Pour l'énoncer, on fixe d'abord quelques notations. Soit $p$ un nombre premier. On fixe deux extensions finies $L$ et $K$ de $\mathbb{Q}_{p}$ contenues dans $\overline{\mathbb{Q}}_{p}$ telles que $\left[L: \mathbb{Q}_{p}\right]=\left|\operatorname{Hom}_{\mathbb{Q}_{p}}(L, K)\right|$, et aussi une extension finie galoisienne $L^{\prime}$ de $L$ telle que $\left[L_{0}^{\prime}: \mathbb{Q}_{p}\right]=\left|\operatorname{Hom}_{\mathbb{Q}_{p}}\left(L_{0}^{\prime}, K\right)\right|$ où $L_{0}^{\prime}$ désigne le sous-corps non-ramifié maximal de $L^{\prime}$. On note $W\left(\overline{\mathbb{Q}}_{p} / L\right)$ (resp. $W\left(\overline{\mathbb{Q}}_{p} / L^{\prime}\right)$ ) le groupe de Weil de $L$ (resp. $L^{\prime}$ ).

On considère les deux catégories suivantes (cf. §2.2) :

(i) $\mathrm{WD}_{L^{\prime} / L}$ : la catégorie des $K$-représentations $(r, N, V)$ de dimension finie du groupe de Weil-Deligne de $L$ telles que la restriction de $r$ à $W\left(\overline{\mathbb{Q}}_{p} / L^{\prime}\right)$ est non-ramifiée;

(ii) $\mathrm{MOD}_{L^{\prime} / L}$ : la catégorie des $(\varphi, N)$-modules étales sur $L_{0}^{\prime} \otimes_{\mathbb{Q}_{p}} K$ munis d'une action de $\operatorname{Gal}\left(L^{\prime} / L\right)$ vérifiant quelques conditions usuelles.

On sait qu'il existe deux foncteurs (non canoniques)

$$
\mathrm{WD}: \mathrm{MOD}_{L^{\prime} / L} \rightarrow \mathrm{WD}_{L^{\prime} / L}, \quad \mathrm{MOD}: \mathrm{WD}_{L^{\prime} / L} \rightarrow \mathrm{MOD}_{L^{\prime} / L}
$$

qui sont inverses l'un de l'autre (cf. §2.2).

Rappelons que (cf. [1]) pour un objet $\left(\varphi, N, \operatorname{Gal}\left(L^{\prime} / L\right), D\right)$ de $\mathrm{MOD}_{L^{\prime} / L}$ on peut définir un entier $t_{N}(D)$ et, si de plus on se donne d'une filtration décroissante exhaustive séparée sur $D_{L^{\prime}}=L^{\prime} \otimes_{L_{0}^{\prime}} D$ qui est stable sous l'action de $\operatorname{Gal}\left(L^{\prime} / L\right)$, on a un autre entier $t_{H}\left(D_{L^{\prime}}\right)$. Par définition cette filtration est dite admissible si $t_{H}\left(D_{L^{\prime}}\right)=t_{N}(D)$ et si $t_{H}\left(D_{L^{\prime}}^{\prime}\right) \leq t_{N}\left(D^{\prime}\right)$ pour tout sous-objet $D^{\prime}$ de $D$ où $D^{\prime}$ est muni de la filtration induite. Pour tout $\sigma: L \hookrightarrow K$, on pose

$$
D_{L^{\prime}, \sigma}=D_{L^{\prime}} \otimes_{L^{\prime} \otimes_{\mathbb{Q}_{p}} K}\left(L^{\prime} \otimes_{L, \sigma} K\right) .
$$

On a un isomorphisme naturel $D_{L^{\prime}} \simeq \prod_{\sigma: L \hookrightarrow K} D_{L^{\prime}, \sigma}$.

Si $(r, N, V) \in \mathrm{WD}_{L^{\prime} / L}$, on note $(r, N, V)^{\mathrm{ss}} \in \mathrm{WD}_{L^{\prime} / L}$ sa $F$-semisimplification (cf. [7], $\S 8.5)$.

On fixe un entier $d \geq 1$ et :

(i) un objet $(r, N, V)$ de $\mathrm{WD}_{L^{\prime} / L}$ de dimension $d+1$ et tel que $r$ est semi-simple;

(ii) pour tout $\sigma: L \hookrightarrow K$, un ensemble de $d+1$ entiers $i_{1, \sigma}<\cdots<i_{d+1, \sigma}$.

Posons $a_{j, \sigma}=-i_{d+2-j, \sigma}-(j-1)$ pour tout $\sigma$ et $j \in\{1, \ldots, d+1\}$. D'une part, à $(r, N, V)$ on associe une représentation lisse $\pi$ de $\mathrm{GL}_{d+1}(L)$ par la correspondance de Langlands locale modifiée et un $(\varphi, N)$-module sur $L_{0}^{\prime} \otimes_{\mathbb{Q}_{p}} K$ muni d'une action de $\operatorname{Gal}\left(L^{\prime} / L\right)$ (cf. $\left.\S 2.2\right)$. D'autre part, à $\left\{a_{j, \sigma}\right\}_{j, \sigma}$ on associe une représentation $\mathbb{Q}_{p}$-rationnelle sur $K$ de $\mathrm{GL}_{d+1}(L)$, notée $\rho$.

Conjecture 1.1. ([3], Conjecture 4.3) Les deux conditions suivantes sont équivalentes :

(i) $\rho \otimes \pi$ admet une norme invariante;

(ii) Il existe un objet $\left(\varphi, N, \operatorname{Gal}\left(L^{\prime} / L\right), D\right)$ dans $\mathrm{MOD}_{L^{\prime} / L}$ tel que :

$$
W D\left(\varphi, N, \operatorname{Gal}\left(L^{\prime} / L\right), D\right)^{\mathrm{ss}}=(r, N, V),
$$

et une filtration admissible $\left(\mathrm{Fil}^{i} D_{L^{\prime}, \sigma}\right)_{i, \sigma}$ stable par $\operatorname{Gal}\left(L^{\prime} / L\right)$ sur $D_{L^{\prime}}$ telle que

$$
\mathrm{Fil}^{i} D_{L^{\prime}, \sigma} / \mathrm{Fil}^{i+1} D_{L^{\prime}, \sigma} \neq 0 \Leftrightarrow i \in\left\{i_{1, \sigma}, \ldots, i_{d+1, \sigma}\right\} .
$$


On renvoie le lecteur à [3], $\S 1$ pour l'introduction de la conjecture. Certains résultats sur cette conjecture sont connus :

- si $(r, N, V)$ est absolument irréductible (alors $N=0)$, la conjecture est vraie ([3], Theorem 5.2);

- si $(r, N, V)$ est absolument indécomposable, alors la condition (ii) est vraie si et seulement si le caractère central de $\rho \otimes \pi$ est unitaire; en particulier, on a (i) entraîne (ii) ([3], Proposition 5.3);

- si $(r, N, V)$ est tel que $N=0$ et $r$ est non-ramifiée et scindée sur $K$, alors (i) entraîne (ii) ([3], Theorem 5.6);

- si $L=L^{\prime}=\mathbb{Q}_{p}$ et $d=1$, la conjecture est vraie ([1], [6]).

Dans cet article, nous montrons le théorème suivant :

Théorème 1.2. Supposons $K$ suffisamment gros tel que l'on a

$$
(r, N, V)=\bigoplus_{i=1}^{s}\left(r_{i}, N_{i}, V_{i}\right)
$$

avec les $\left(r_{i}, N_{i}, V_{i}\right)$ absolument indécomposables de dimension $d_{i}$. On considère les quatre conditions suivantes :

(i) $\rho \otimes \pi$ admet une norme invariante.

(ii) Il existe un objet $\left(\varphi, N, \operatorname{Gal}\left(L^{\prime} / L\right), D\right)$ dans $\mathrm{MOD}_{L^{\prime} / L}$ tel que :

$$
W D\left(\varphi, N, \operatorname{Gal}\left(L^{\prime} / L\right), D\right)^{\mathrm{ss}}=(r, N, V),
$$

et une filtration admissible $\left(\mathrm{Fil}^{i} D_{L^{\prime}, \sigma}\right)_{i, \sigma}$ stable par $\operatorname{Gal}\left(L^{\prime} / L\right) \times \operatorname{Gal}\left(K / \mathbb{Q}_{p}\right)$ sur $D_{L^{\prime}}$ telle que

$$
\mathrm{Fil}^{i} D_{L^{\prime}, \sigma} / \mathrm{Fil}^{i+1} D_{L^{\prime}, \sigma} \neq 0 \Leftrightarrow i \in\left\{i_{1, \sigma}, \ldots, i_{d+1, \sigma}\right\} .
$$

(iii) Posons $\left(\varphi_{i}, N_{i}, \operatorname{Gal}\left(L^{\prime} / L\right), D_{i}\right)=\operatorname{MOD}\left(r_{i}, N_{i}, V_{i}\right)$. Pour une permutation convenable $\nu$ de $\{1, \ldots, s\}$ (voir §2.4), les inégalités suivantes sont vérifiées :

$$
\begin{gathered}
{[K: L] \sum_{j=1}^{d_{\nu(1)}} \sum_{\sigma} i_{j, \sigma} \leq t_{N}\left(D_{\nu(1)}\right),} \\
\vdots \\
{[K: L] \sum_{j=1}^{d_{\nu(1)}+\cdots+d_{\nu(s-1)}} \sum_{\sigma} i_{j, \sigma} \leq \sum_{i=1}^{s-1} t_{N}\left(D_{\nu(i)}\right),} \\
{[K: L] \sum_{j=1}^{d+1} \sum_{\sigma} i_{j, \sigma}=\sum_{i=1}^{s} t_{N}\left(D_{\nu(i)}\right)=t_{N}(D) .}
\end{gathered}
$$

(iv) Le caractère central de $\rho \otimes \pi$ est unitaire et la condition d'Emerton est satisfaite

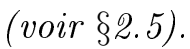

Alors, on a les implications et équivalences :

$$
(i) \Rightarrow(i i) \Leftrightarrow(i i i) \Leftrightarrow(i v) .
$$


Remarque 1.3. L'existence de la permutation $\nu$ dans (iii) et l'équivalence entre (ii) et (iii) sont la réponse de [3], Remark 5.7.

Comme conséquence, on obtient (cf. corollaire 2.16)

Corollaire 1.4. Avec les notations comme dans la conjecture 1.1, on a l'implication

$$
(i) \Rightarrow(i i)
$$

Voici le plan de l'article :

On commence par rappeler certaines des constructions et notations dans [3] pour pouvoir énoncer la conjecture précédente et le théorème principal (§2). Les autres chapitres sont consacrés à prouver notre théorème. Plus précisément, on prouve

$$
(i) \Rightarrow(i v) \Leftrightarrow(i i i) \Leftrightarrow(i i)
$$

L'implication $(i) \Rightarrow(i v)$ est en fait un lemme d'Emerton (cf. lemme 2.9) et $(i i) \Rightarrow(i i i)$ se déduit de la définition d'admissibilité. Au $\S 3$, on montre $(i i i) \Leftrightarrow(i v)$ en exprimant la condition d'Emerton explicitement par la théorie de Bernstein-Zelevinsky.

$\mathrm{Au} \S 4$, on montre $(\mathrm{iii}) \Rightarrow(\mathrm{ii})$. Cette partie, bien que constituée d'algèbre (semi)linéaire, est la plus technique de l'article. L'idée de la démonstration est comme suit : Après l'étude de quelques exemples, on définit d'abord l'objet $\left(\varphi, N, \operatorname{Gal}\left(L^{\prime} / L\right), D\right.$ ) (essentiellement l'opérateur $\varphi$ ). Au $\S 4.2$ on introduit un certain ensemble fini de sous-objets de $D$, noté $S$, et on munit $D_{L^{\prime}}$ d'une filtration convenable ( $\left.\$ 4.3\right)$ telle que pour tout $D^{\prime} \in S$ on a

$$
t_{H}\left(D_{L^{\prime}}^{\prime}\right)=\sum_{j=1}^{\operatorname{rg} D^{\prime}} \sum_{\sigma} i_{j, \sigma},
$$

et par conséquent $t_{H}\left(D_{L^{\prime}}^{\prime}\right) \leq t_{N}\left(D^{\prime}\right)$. On montre que cette filtration est admissible. Pour cela, soit $D^{\prime}$ un sous-objet quelconque de $D$, on lui associe une suite de sous-objets $E_{i} \in S$

$$
0=E_{0} \subsetneq E_{1} \subsetneq \cdots \subsetneq E_{m} \subsetneq E_{m+1}=D,
$$

qui est la plus proche de $D^{\prime}$ au sens où si $L \in S$ est de même rang que $E_{i}$, alors

$$
\operatorname{rg}_{L_{0}^{\prime} \otimes_{\mathbb{Q}_{p}} K}\left(D^{\prime} \cap L\right) \leq \operatorname{rg}_{L_{0}^{\prime} \otimes_{\mathbb{Q}_{p}} K}\left(D^{\prime} \cap E_{i}\right)
$$

D'autre part, si on pose $c_{i}=\operatorname{rg}\left(D^{\prime} \cap E_{i}\right)-\operatorname{rg}\left(D^{\prime} \cap E_{i-1}\right)\left(\operatorname{sur} L_{0}^{\prime} \otimes_{\mathbb{Q}_{p}} K\right)$ et

$$
\Omega=\left\{j \in \mathbb{N} \mid \exists i \text { tel que } \operatorname{rg} E_{i}-c_{i}+1 \leq j \leq \operatorname{rg} E_{i}\right\},
$$

alors on a $t_{H}\left(D_{L^{\prime}}^{\prime}\right) \leq[K: L] \sum_{j \in \Omega} \sum_{\sigma} i_{j, \sigma}$. Enfin on compare $[K: L] \sum_{j \in \Omega} \sum_{\sigma} i_{j, \sigma}$ et $t_{N}\left(D^{\prime}\right)$ en utilisant un lemme combinatoire, et on en déduit que $t_{H}\left(D_{L^{\prime}}^{\prime}\right) \leq t_{N}\left(D^{\prime}\right)$ (cf. le corollaire 4.11).

Remerciement. Ce travail s'est accompli sous la direction de C. Breuil. Je le remercie pour avoir partagé avec moi ses idées et ses connaissances et pour toutes ses remarques. 


\section{Rappels et notations}

\subsection{Notations générales}

Soit $p$ un nombre premier. On fixe une clôture algébrique $\overline{\mathbb{Q}}_{p}$ de $\mathbb{Q}_{p}$, et aussi deux extensions finies $L$ et $K$ de $\mathbb{Q}_{p}$ contenues dans $\overline{\mathbb{Q}}_{p}$ telles que $\left[L: \mathbb{Q}_{p}\right]=\left|\operatorname{Hom}_{\mathbb{Q}_{p}}(L, K)\right|$. On note $\operatorname{Gal}\left(\overline{\mathbb{Q}}_{p} / L\right)$ le groupe de Galois de $L$ et $W\left(\overline{\mathbb{Q}}_{p} / L\right)$ son groupe de Weil (qui est

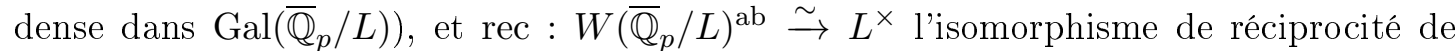
telle sorte que les Frobenius arithmétiques s'envoient sur les inverses des uniformisantes. Posons $k_{L}$ le corps résiduel de $L$ et $q=p^{f}=\left|k_{L}\right|$. On note $L_{0}=\operatorname{Frac}\left(W\left(\mathbb{F}_{q}\right)\right)$ le souscorps non-ramifié maximal de $L$ et $\varphi_{0}$ le Frobenius sur $L_{0}$. On note val $\operatorname{va}_{p}$ la valuation $p$-adique sur $\overline{\mathbb{Q}}_{p}$ normalisée par $\operatorname{val}_{p}(p)=1$ et on pose $|x|_{p}=p^{-\operatorname{val}_{p}(x)}$; de même on pose $\operatorname{val}_{L}(x)=e \operatorname{val}_{p}(x)$ et $|x|_{L}=q^{-\operatorname{val}_{L}(x)}$, où $e=\left[L: \mathbb{Q}_{p}\right] / f$ est l'indice de ramification de $L \operatorname{sur} \mathbb{Q}_{p}$.

Fixons $L^{\prime}$ une extension finie galoisienne de $L$. On définit $L_{0}^{\prime}, k_{L^{\prime}}, f^{\prime}, \varphi_{0}^{\prime}, \operatorname{val}_{L^{\prime}}$ comme ci-dessus. On suppose que $\left[L_{0}^{\prime}: \mathbb{Q}_{p}\right]=\left|\operatorname{Hom}_{\mathbb{Q}_{p}}\left(L_{0}^{\prime}, K\right)\right|$.

Si $\mathbb{G}$ est un groupe réductif défini sur $\mathbb{Q}_{p}$ et si $G=\mathbb{G}(L)$ est le groupe des $L$ points rationnels de $\mathbb{G}$, on note $\operatorname{Rep} G$ la catégorie des $\overline{\mathbb{Q}}_{p}$-représentations lisses de $G$ et $\operatorname{Irr} G$ la sous-catégorie formée des représentations irréductibles. Soit $P$ un sous-groupe parabolique de $G$ avec $M$ son quotient de Levi et $N$ son radical unipotent. On définit les foncteurs suivants :

$$
\begin{gathered}
\operatorname{ind}_{P}^{G}: \operatorname{Ind}_{P}^{G}: \operatorname{Rep} M \rightarrow \operatorname{Rep} G, \\
r_{P}^{G}: \operatorname{Rep} G \rightarrow \operatorname{Rep} M .
\end{gathered}
$$

(a) Soit $(\sigma, W) \in \operatorname{Rep} G$, notons $\operatorname{ind}_{P}^{G} \sigma$ l'espace des fonctions $f: G \rightarrow W$ telles que

- $f(n m g)=\sigma(m) f(g)$, si $n \in N, m \in M$ et $g \in G$,

- $f$ est invariante à droite par un sous-groupe ouvert de $G$.

Le groupe $G$ opère par translation à droite et on obtient une représentation lisse de $M$. On pose $\operatorname{Ind}_{P}^{G} \sigma=\operatorname{ind}_{P}^{G}\left(\sigma \delta_{P}^{1 / 2}\right)$, où $\delta_{P}$ est le caractère module de $P$, c'est-à-dire, le caractère de $M=P / N$ donné par : $\delta_{P}(m)=\left[m N_{0} m^{-1}: N_{0}\right]$ pour un arbitraire sous-groupe ouvert compact $N_{0}$ de $N$.

(b) Soit $(\pi, V) \in \operatorname{Rep} G$, on note $V_{N}$ le quotient de $V$ par le sous-espace $V(N)$ engendré par les éléments $\pi(n) x-x(n \in N, x \in V)$. On définit $\left(r_{P}^{G}(\pi), V_{N}\right) \in \operatorname{Rep} M$ par

$$
r_{P}^{G}(m)(v+V(N))=\delta_{P}^{-1 / 2}(m)(\pi(m) v+V(N)), \quad m \in M, v \in V .
$$

\subsection{Rappels de quelques constructions}

Rappelons que dans [3], §4, sont définies deux catégories $\mathrm{MOD}_{L^{\prime} / L}$ et $\mathrm{WD}_{L^{\prime} / L}$ :

(i) $\mathrm{WD}_{L^{\prime} / L}$ : la catégorie des $K$-représentations $(r, N, V)$ du groupe de Weil-Deligne de $L([7], \S 8)$ sur un $K$-espace vectoriel $V$ de dimension finie telles que la restriction de $r$ à $W\left(\overline{\mathbb{Q}}_{p} / L^{\prime}\right)$ est non-ramifiée;

(ii) $\mathrm{MOD}_{L^{\prime} / L}$ (ou $\mathrm{MOD}_{L^{\prime} / L, K}$ s'il y a risque de confusion sur $K$ ) : la catégorie des quadruples $\left(\varphi, N, \operatorname{Gal}\left(L^{\prime} / L\right), D\right)$ constitués par :

- un $L_{0}^{\prime} \otimes_{\mathbb{Q}_{p}} K$-module D libre de rang fini;

- un Frobenius $\varphi: D \rightarrow D$, c'est-à-dire une bijection $\varphi$ tel que $\varphi((l \otimes k) \cdot d)=$ $\left(\varphi_{0}^{\prime}(l) \otimes k\right) \cdot \varphi(d)$, pour $l \in L_{0}^{\prime}, k \in K, d \in D$; 
- un endomorphisme $L_{0}^{\prime} \otimes_{\mathbb{Q}_{p}} K$-linéaire $N: D \rightarrow D$ tel que $N \varphi=p \varphi N$;

- une action de $\operatorname{Gal}\left(L^{\prime} / L\right)$ sur $D$ commutant avec celles de $\varphi$ et $N$, telle que $g((l \otimes$ $k) \cdot d)=(g(l) \otimes k) \cdot g(d)$, pour $g \in \operatorname{Gal}\left(L^{\prime} / L\right), l \in L_{0}^{\prime}, k \in K, d \in D$.

Rappelons aussi que dans [3], $\S 4$ (ou [12]), sont définis un foncteur

$$
\mathrm{WD}: \mathrm{MOD}_{L^{\prime} / L} \rightarrow \mathrm{WD}_{L^{\prime} / L}
$$

et un quasi-inverse MOD de WD. Ces foncteurs induisent une équivalence entre les deux catégories. On rappelle la construction dans la suite.

- Soit $\left(\varphi, N, \operatorname{Gal}\left(L^{\prime} / L\right), D\right)$ un objet de $\operatorname{MOD}_{L^{\prime} / L}$. Choisissons un plongement $\sigma_{0}^{\prime}$ : $L_{0}^{\prime} \hookrightarrow K$ et posons $V=D_{\sigma_{0}^{\prime}}$. Alors $N$ induit un endomorphisme $K$-linéaire nilpotent sur $V$ que l'on note encore par $N$. Si $w \in W\left(\overline{\mathbb{Q}}_{p} / L\right)$, on définit $r(w)=$ $\bar{w} \circ \varphi^{-\alpha(w)}$ où $\bar{w}$ désigne l'image de $w$ dans $\operatorname{Gal}\left(L^{\prime} / L\right)$ et $\alpha(w) \in f \mathbb{Z}$ est l'unique entier tel que l'action induite de $w$ sur $\overline{\mathbb{F}}_{p}$ soit la $\alpha(w)$-puissance du Frobenius arithmétique $x \mapsto x^{p}$. On vérifie que $r(w)$ est $L_{0}^{\prime} \otimes_{\mathbb{Q}_{p}} K$-linéaire, et donc induit un morphisme $K$-linéaire $r(w): V \rightarrow V$. Ceci définit un objet de $\mathrm{WD}_{L^{\prime} / L}$. Remarquons que la représentation $(r, N, V)$ est indépendante du choix de $\sigma_{0}^{\prime}$ mais à isomorphisme non canonique près (cf. [2], lemme 2.2.1.2).

- Soit $(r, N, V)$ un objet de $\mathrm{WD}_{L^{\prime} / L}$ et choisissons un plongement $\sigma_{0}^{\prime}: L_{0}^{\prime} \hookrightarrow K$. On pose

$$
D=\bigoplus_{n=0}^{f^{\prime}-1} V_{\sigma_{0}^{\prime} \circ \varphi_{0}^{\prime-n}}
$$

où $V_{\sigma_{0}^{\prime} \circ \varphi_{0}^{\prime-n}}=V$ est muni de l'action de $L_{0}^{\prime}$ via $\sigma_{0}^{\prime} \circ \varphi_{0}^{\prime-n}$, ce qui fait de $D$ un $L_{0}^{\prime} \otimes_{\mathbb{Q}_{p}} K$-module libre. On définit $\varphi, N$ par

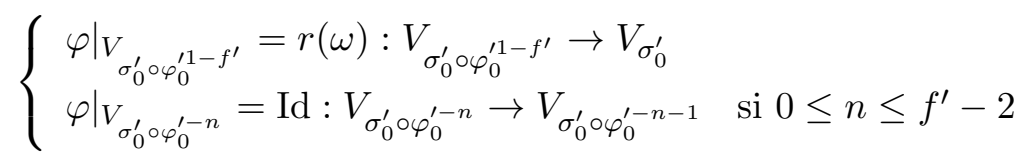

où $\omega$ est n'importe quel Frobenius géométrique dans $W\left(\overline{\mathbb{Q}}_{p} / L^{\prime}\right)$, et

$$
\left\{\begin{array}{l}
\left.N\right|_{V_{\sigma_{0}^{\prime}}}=\left.N\right|_{V}: V_{\sigma_{0}^{\prime}} \rightarrow V_{\sigma_{0}^{\prime}} \\
\left.N\right|_{V_{\sigma_{0}^{\prime} \circ \varphi_{0}^{\prime-n}}}=\left.p^{n} \varphi^{n} \circ N\right|_{V} \circ \varphi^{-n}: V_{\sigma_{0}^{\prime} \circ \varphi_{0}^{\prime-n}} \rightarrow V_{\sigma_{0}^{\prime} \circ \varphi_{0}^{\prime-n}} \text { si } 1 \leq n \leq f^{\prime}-1 .
\end{array}\right.
$$

Finalement, pour $g \in \operatorname{Gal}\left(L^{\prime} / L\right)$, soit $w \in W\left(\overline{\mathbb{Q}}_{p} / L\right)$ un relèvement de $g$, on définit l'action de $g$ sur $V_{\sigma_{0}^{\prime} \circ \varphi_{0}^{\prime-n}}$ par

$$
g=r(w) \circ \varphi^{\alpha(w)}: V_{\sigma_{0}^{\prime} \circ \varphi_{0}^{\prime-n}} \rightarrow V_{\sigma_{0}^{\prime} \circ \varphi_{0}^{\prime-n-\alpha(w)}},
$$

où si $n+\alpha(w)>f^{\prime}-1$ ou $n+\alpha(w)<0$, on regarde $V_{\sigma_{0}^{\prime} \circ \varphi_{0}^{\prime-n-\alpha(w)}}$ comme $V_{\sigma_{0}^{\prime} \circ \varphi_{0}^{\prime-k}}$ où $k$ est l'unique entier tel que

$$
0 \leq k \leq f^{\prime}-1 \text { et } n+\alpha(w) \equiv k\left(\bmod f^{\prime}\right) .
$$

On vérifie que $\left(\varphi, N, \operatorname{Gal}\left(L^{\prime} / L\right), D\right)$ ainsi défini est un objet de $\operatorname{MOD}_{L^{\prime} / L}$. 
Remarque 2.1. Soit $\left(\varphi, N, \operatorname{Gal}\left(L^{\prime} / L\right), D\right)$ un objet de $\mathrm{MOD}_{L^{\prime} / L}$ qui est absolument irréductible (donc $N=0$ ). Comme $\varphi^{f^{\prime}}$ est $L_{0}^{\prime} \otimes_{\mathbb{Q}_{p}} K$-linéaire et commute avec $\operatorname{Gal}\left(L^{\prime} / L\right)$ et $\varphi$, on voit que $\varphi^{f^{\prime}}$ est scalaire à valeur dans $K^{\times}$.

Maintenant, soit $\left(\varphi, N, \operatorname{Gal}\left(L^{\prime} / L\right), D\right)$ un objet de $\mathrm{MOD}_{L^{\prime} / L}$. On définit

$$
t_{N}(D)=\frac{1}{\left[L: L_{0}\right] f^{\prime}} \operatorname{val}_{L}\left(\operatorname{det}_{L_{0}^{\prime}}\left(\left.\varphi^{f^{\prime}}\right|_{D}\right)\right)
$$

Posons $D_{L^{\prime}}=D \otimes_{L_{0}^{\prime}} L^{\prime}$ et pour $\sigma: L \hookrightarrow K$,

$$
D_{L^{\prime}, \sigma}=D_{L^{\prime}} \otimes_{L^{\prime} \otimes_{\mathbb{Q}_{p}} K}\left(L^{\prime} \otimes_{L, \sigma} K\right) .
$$

On a alors $D_{L^{\prime}} \simeq \prod_{\sigma: L \hookrightarrow K} D_{L^{\prime}, \sigma}$. Donc la donnée d'une filtration décroissante exhaustive séparée par des sous- $L^{\prime} \otimes_{\mathbb{Q}_{p}} K$-modules $\left(\mathrm{Fil}^{i} D_{L^{\prime}}\right)_{i}$ (pas forcément libres) stables sous l'action de $\operatorname{Gal}\left(L^{\prime} / L\right)$ équivaut à la donnée, pour tout $i \in \mathbb{Z}$ et tout $\sigma: L \rightarrow K$, d'un sous$L^{\prime} \otimes_{L, \sigma} K$-module $\mathrm{Fil}^{i} D_{L^{\prime}, \sigma}$ de $D_{L^{\prime}, \sigma}$, qui est stable par $\operatorname{Gal}\left(L^{\prime} / L\right)$ (donc nécessairement libre), et vérifie $\mathrm{Fil}^{i+1} D_{L^{\prime}, \sigma} \subset \mathrm{Fil}^{i} D_{L^{\prime}, \sigma}$ pour tout $i$ et $\sigma$, et $\cup_{i \in \mathbb{Z}} \mathrm{Fil}^{i} D_{L^{\prime}, \sigma}=D_{L^{\prime}, \sigma}$, $\cap_{i \in \mathbb{Z}} \mathrm{Fil}^{i} D_{L^{\prime}, \sigma}=0$ pour tout $\sigma$. Soit $\left(\mathrm{Fil}^{i} D_{L^{\prime}, \sigma}\right)_{i, \sigma}$ une telle filtration. On définit

$$
t_{H}\left(D_{L^{\prime}}\right)=\sum_{i \in \mathbb{Z}} \sum_{\sigma} i \operatorname{dim}_{L^{\prime}}\left(\mathrm{Fil}^{i} D_{L^{\prime}, \sigma} / \mathrm{Fil}^{i+1} D_{L^{\prime}, \sigma}\right) .
$$

La filtration est dite admissible si $t_{H}\left(D_{L^{\prime}}\right)=t_{N}(D)$ et si pour tout sous- $L_{0}^{\prime}$-espace vectoriel $D^{\prime}$ de $D$ stable par $\varphi$ et $N$, on a $t_{H}\left(D_{L^{\prime}}^{\prime}\right) \leq t_{N}\left(D^{\prime}\right)$, où $D_{L^{\prime}}^{\prime}$ est muni de la filtration induite par $D_{L^{\prime}}$. Par [2], proposition 3.1.1.5 et [11], proposition 4.4.9, pour que la filtration soit admissible, il suffit de vérifier $t_{H}\left(D_{L^{\prime}}\right)=t_{N}(D)$ et l'inégalité $t_{H}\left(D_{L^{\prime}}^{\prime}\right) \leq t_{N}\left(D^{\prime}\right)$ pour les $L_{0}^{\prime} \otimes_{\mathbb{Q}_{p}} K$-modules $D^{\prime}$ stables par $\varphi, N$ et $\operatorname{Gal}\left(L^{\prime} / L\right)$ (nécessairement libre), c'est-à-dire les sous-objets de $D$ dans $\operatorname{MOD}_{L^{\prime} / L}$.

Si $n \in \mathbb{N}$, on pose $G_{n}=G L_{n}(L)$ et $G=G_{d+1}$ où $d \geq 1$ est un entier fixé. Une partition de l'entier $n$ est une suite $\alpha=\left(n_{1}, \ldots, n_{r}\right)$ d'entiers positifs tels que $n=n_{1}+\cdots+n_{r}$. Étant donné une telle partition, on note $P_{\alpha}$ le groupe formé des matrices inversibles triangulaires supérieures par blocs, c'est-à-dire,

$$
P_{\alpha}=\left(\begin{array}{cccc}
G_{n_{1}} & * & * & * \\
& G_{n_{2}} & * & * \\
& & \ddots & \ldots \\
& & & G_{n_{r}}
\end{array}\right) .
$$

On note $N_{\alpha}=N_{P_{\alpha}}$ son radical unipotent et $M_{\alpha}=M_{P_{\alpha}}$ son quotient de Levi. Les groupes $P_{\alpha}$ seront appelés sous-groupe paraboliques standards de $G_{n}$. Si $\alpha$ et $\beta$ sont deux partitions de $n$, on dit que $\beta$ est une sous-partition de $\alpha$ si $M_{\beta} \subseteq M_{\alpha}$ (notation : $\beta \leq \alpha$ ).

On fixe un choix de $q^{1 / 2}$ dans $\overline{\mathbb{Q}}_{p}$. À une représentation $(r, N, V)$ de dimension $d+1$ telle que $r$ soit semi-simple, la correspondance de Langlands locale permet d'associer une représentation irréductible lisse $\pi^{\text {unit }}$ de $G$ sur $\overline{\mathbb{Q}}_{p}$ normalisée de sorte que le caractère central de $\pi^{\text {unit }}$ soit $\operatorname{det}(r, N, V) \circ \operatorname{rec}^{-1}$. Notons que la définition dépend du choix de $q^{1 / 2}$. Dans [3], cette correspondance est modifiée comme suit : 
- si $\pi^{\text {unit }}$ est générique (cf. [14], §2.3), alors $\pi^{\text {unit }} \otimes_{\overline{\mathbb{Q}}_{p}}|\operatorname{det}|_{L}^{-d / 2}$ admet un unique modèle sur $K$ qui ne dépend pas du choix de $q^{1 / 2}$; on le note $\pi$;

- si $\pi^{\text {unit }}$ n'est pas générique, elle s'écrit comme l'unique quotient d'une induction parabolique :

$$
\operatorname{Ind}_{P_{\alpha}}^{G} L\left(b_{1}, \tau_{1}\right) \otimes \cdots \otimes L\left(b_{s}, \tau_{s}\right)
$$

où $\alpha=\left(b_{1} n_{1}, \ldots, b_{s} n_{s}\right)$ est une partition de $d+1$, les $\tau_{i}$ sont des représentations irréductibles cuspidales de $\mathrm{GL}_{n_{i}}(L)$, les $L\left(b_{i}, \tau_{i}\right)$ sont des Steinberg généralisées, c'est-à-dire, $L\left(b_{i}, \tau_{i}\right)$ est l'unique quotient irréductible de

$$
\operatorname{Ind}_{P_{\alpha_{i}}}^{G_{b_{i} n_{i}}} \tau_{i} \otimes \tau_{i}|\operatorname{det}|_{L} \otimes \cdots \otimes \tau_{i}|\operatorname{det}|_{L}^{b_{i}-1}
$$

où $\alpha_{i}$ est la partition $\left(n_{i}, \ldots, n_{i}\right)$ de $b_{i} n_{i}$ (cf. [5], §3.1). On a le résultat suivant :

Lemme 2.2. ([3], Lemma 4.2) La représentation

$$
\left(\operatorname{Ind}_{P_{\alpha}}^{G} L\left(b_{1}, \tau_{1}\right) \otimes \cdots \otimes L\left(b_{s}, \tau_{s}\right)\right) \otimes_{\overline{\mathbb{Q}}_{p}}|\operatorname{det}|_{L}^{-d / 2}
$$

admet un modèle unique sur $K$ qui ne dépend pas du choix de $q^{1 / 2}$. On le note $\pi$.

Si on suppose que $K$ est gros au sens où tous les $L\left(b_{i}, \tau_{i}\right)$ sont fixées par $\operatorname{Gal}\left(\overline{\mathbb{Q}}_{p} / K\right)$, alors la définition de $\pi$ est plus directe. En fait, si on pose

$$
\mathcal{L}\left(b_{i}, \tau_{i}\right)=L\left(b_{i}, \tau_{i}\right) \otimes_{\overline{\mathbb{Q}}_{p}}|\operatorname{det}|_{L}^{\left(1-b_{i} n_{i}\right) / 2},
$$

alors cette $\mathcal{L}\left(b_{i}, \tau_{i}\right)$ ainsi définie ne dépend pas du choix de $q^{1 / 2}$, et est fixée par $\operatorname{Gal}\left(\overline{\mathbb{Q}}_{p} / K\right)$. D'après [5], proposition 3.2, elle admet un modèle unique qui est défini sur $K$ et on le note $\pi_{i}$. Comme on peut récrire la représentation originale sous la forme

$$
\operatorname{ind}_{P_{\alpha}}^{G} \mathcal{L}\left(b_{1}, \tau_{1}\right) \otimes \mathcal{L}\left(b_{2}, \tau_{2}\right)|\operatorname{det}|_{L}^{-b_{1} n_{1}} \otimes \cdots \otimes \mathcal{L}\left(b_{s}, \tau_{s}\right)|\operatorname{det}|_{L}^{-\sum_{j=1}^{s-1} b_{j} n_{j}},
$$

on obtient le modèle $\pi$ en posant

$$
\pi=\operatorname{ind}_{P_{\alpha}}^{G} \pi_{1} \otimes \pi_{2}|\operatorname{det}|_{L}^{-b_{1} n_{1}} \otimes \cdots \otimes \pi_{s}|\operatorname{det}|_{L}^{-\sum_{j=1}^{s-1} b_{j} n_{j}} .
$$

\subsection{Rappels sur la conjecture et compléments}

On fixe :

(i) un objet $(r, N, V)$ de $\mathrm{WD}_{L^{\prime} / L}$ de dimension $d+1$ tel que $r$ soit semi-simple;

(ii) pour tout $\sigma: L \hookrightarrow K$, un ensemble de $d+1$ entiers $i_{1, \sigma}<\cdots<i_{d+1, \sigma}$.

À $(r, N, V)$ comme dans (i) on associe une représentation lisse $\pi$ comme au §2.2. Pour des $i_{j, \sigma}$ comme dans (ii), on pose

$$
a_{j, \sigma}=-i_{d+2-j, \sigma}-(j-1),
$$

et on note $\rho$ l'unique représentation $\mathbb{Q}_{p}$-rationnelle de $G$ dont le plus haut poids est $\psi: \operatorname{diag}\left(x_{1}, \ldots, x_{d+1}\right) \mapsto \prod_{j=1}^{d+1} \prod_{\sigma: L \hookrightarrow K} x_{j}^{a_{j, \sigma}}$ vis-à-vis du sous-groupe des matrices triangulaires inférieures (cf. [3], §2). $\S 8.5)$.

Si $(r, N, V) \in \mathrm{WD}_{L^{\prime} / L}$, on note $(r, N, V)^{\mathrm{ss}} \in \mathrm{WD}_{L^{\prime} / L}$ sa $F$-semisimplification ([7],

La conjecture ci-dessous est proposée dans [3], Conjecture 4.3 : 
Conjecture 2.3. Les deux conditions suivantes sont équivalentes :

(i) $\rho \otimes \pi$ admet une norme invariante;

(ii) il existe un objet $\left(\varphi, N, \operatorname{Gal}\left(L^{\prime} / L\right), D\right)$ dans $\mathrm{MOD}_{L^{\prime} / L}$ tel que :

$$
W D\left(\varphi, N, \operatorname{Gal}\left(L^{\prime} / L\right), D\right)^{\mathrm{ss}}=(r, N, V),
$$

et une filtration admissible $\left(\mathrm{Fil}^{i} D_{L^{\prime}, \sigma}\right)_{i, \sigma}$ stable par $\operatorname{Gal}\left(L^{\prime} / L\right)$ sur $D_{L^{\prime}}$ telle que

$$
\mathrm{Fil}^{i} D_{L^{\prime}, \sigma} / \mathrm{Fil}^{i+1} D_{L^{\prime}, \sigma} \neq 0 \Leftrightarrow i \in\left\{i_{1, \sigma}, \ldots, i_{d+1, \sigma}\right\} .
$$

Proposition 2.4. La condition (ii) ne dépend pas du choix de $L^{\prime}$. Plus précisément, si $L^{\prime \prime}$ est une autre extension finie de $L$ telle que

$$
\left[L_{0}^{\prime \prime}: \mathbb{Q}_{p}\right]=\left|\operatorname{Hom}\left(L_{0}^{\prime \prime}, K\right)\right| \quad \text { et } \quad(r, N, V) \in \mathrm{WD}_{L^{\prime \prime} / L},
$$

alors, (ii) est vraie pour $L^{\prime}$ si et seulement si (ii) est vraie pour $L^{\prime \prime}$.

Démonstration. On peut supposer que $L^{\prime} \subset L^{\prime \prime}$.

(a) Soit $\left(\varphi^{\prime}, N^{\prime}, \operatorname{Gal}\left(L^{\prime} / L\right), D^{\prime}\right)$ un objet de $\mathrm{MOD}_{L^{\prime} / L}$. On pose

$$
D^{\prime \prime}=L_{0}^{\prime \prime} \otimes_{L_{0}^{\prime}} D^{\prime},
$$

et définit $\varphi^{\prime \prime}, N^{\prime \prime}$ et l'action de $\operatorname{Gal}\left(L^{\prime \prime} / L\right)$ naturellement. Alors, on vérifie facilement que $\left(\varphi^{\prime \prime}, N^{\prime \prime}, \operatorname{Gal}\left(L^{\prime \prime} / L\right), D^{\prime \prime}\right)$ est un objet de $\operatorname{MOD}_{L^{\prime \prime} / L}$.

De plus, on suppose que

$$
\mathrm{WD}\left(\varphi^{\prime}, N^{\prime}, \operatorname{Gal}\left(L^{\prime} / L\right), D^{\prime}\right)^{\mathrm{ss}}=(r, N, V)
$$

et qu'il existe une filtration $\left(\mathrm{Fil}^{i} D_{L^{\prime}, \sigma}^{\prime}\right)_{i, \sigma}$ sur $D_{L^{\prime}}^{\prime}$ comme dans (ii). On munit $D^{\prime \prime}$ de la filtration induite : $\mathrm{Fil}^{i} D_{L^{\prime \prime}, \sigma}^{\prime \prime}=L^{\prime \prime} \otimes_{L^{\prime}} \mathrm{Fil}^{i} D_{L^{\prime}, \sigma}^{\prime}$. On vérifie que cette filtration est stable par $\operatorname{Gal}\left(L^{\prime \prime} / L\right)$ et est admissible, en fait on a $t_{N}\left(D^{\prime}\right)=t_{N}\left(D^{\prime \prime}\right)$ et $t_{H}\left(D_{L^{\prime}}^{\prime}\right)=t_{H}\left(D_{L^{\prime \prime}}^{\prime \prime}\right)$. On fixe un plongement $\sigma_{0}^{\prime}: L_{0}^{\prime} \hookrightarrow K$ et $\sigma_{0}^{\prime \prime}: L_{0}^{\prime \prime} \hookrightarrow K$ qui prolonge $\sigma_{0}^{\prime}$. Alors $x \otimes k \mapsto$ $(1 \otimes x) \otimes k$ induit un isomorphisme entre $D_{\sigma_{0}^{\prime}}^{\prime}$ et $D_{\sigma_{0}^{\prime \prime}}^{\prime \prime}$, et cet isomorphisme commute à $r$ et $N$. Donc on a $\operatorname{WD}\left(D^{\prime}\right) \simeq \mathrm{WD}\left(D^{\prime \prime}\right)$.

(b) Soit $\left(\varphi^{\prime \prime}, N^{\prime \prime}, \operatorname{Gal}\left(L^{\prime \prime} / L\right), D^{\prime \prime}\right) \in \mathrm{MOD}_{L^{\prime \prime} / L}$ et supposons qu'il existe une filtration $\left(\mathrm{Fil}^{i} D_{L^{\prime \prime}, \sigma}^{\prime \prime}\right)_{i, \sigma}$ sur $D_{L^{\prime \prime}}^{\prime \prime}$ comme dans (ii). Posons $D^{\prime}=D^{\prime \prime G a l}\left(L^{\prime \prime} / L^{\prime}\right)$ que l'on munit des structures induites : action de $\operatorname{Gal}\left(L^{\prime} / L\right)$, opérateurs $\varphi^{\prime}, N^{\prime}$ et filtration. On vérifie que $\left(\varphi^{\prime}, N, \operatorname{Gal}\left(L^{\prime} / L\right), D^{\prime}\right)$ satisfait à (ii) et on a $L_{0}^{\prime \prime} \otimes_{L_{0}^{\prime}} D^{\prime \prime} \operatorname{Gal}\left(L^{\prime \prime} / L^{\prime}\right)=D^{\prime \prime}$ par Hilbert 90.

Proposition 2.5. Soit $K^{\prime}$ une extension galoisienne finie de $K$ contenue dans $\overline{\mathbb{Q}}_{p}$. Alors

(1) la condition (i) est vraie pour $K$ si et seulement si (i) est vraie pour $K^{\prime}$;

(2) la condition (ii) est vraie pour $K$ si et seulement si (ii) est vraie pour $K^{\prime}$ et la filtration peut être choisie de telle sorte qu'elle soit stable par l'action de $\operatorname{Gal}\left(K^{\prime} / K\right)$.

Démonstration. (1) est immédiat.

(2) La condition est bien nécessaire. Soit $\left(\varphi^{\prime}, N^{\prime}, \operatorname{Gal}\left(L^{\prime} / L\right), D^{\prime}\right)$ un objet de $\mathrm{MOD}_{L^{\prime} / L, K^{\prime}}$ vérifiant (ii) où $D^{\prime}$ est un $L_{0}^{\prime} \otimes_{\mathbb{Q}_{p}} K^{\prime}$-module. Posons $D=D^{\prime \operatorname{Gal}\left(K^{\prime} / K\right)}$ qui est un $L_{0}^{\prime} \otimes_{\mathbb{Q}_{p}} K$ module. Comme $\varphi^{\prime}, N^{\prime}$ et $\operatorname{Gal}\left(L^{\prime} / L\right)$ commutent avec $\operatorname{Gal}\left(K^{\prime} / K\right)$, on obtient un objet 
induit $\left.\left(\varphi, N, \operatorname{Gal}\left(L^{\prime} / L\right), D\right)\right)$ de $\mathrm{MOD}_{L^{\prime} / L, K}$. De plus, puisque la filtration sur $D_{L^{\prime}}^{\prime}$ est stable par $\operatorname{Gal}\left(K^{\prime} / K\right)$, elle induit une filtration sur $D_{L^{\prime}}$ qui est bien admissible. Comme

$$
\mathrm{WD}(D)^{\mathrm{ss}} \otimes_{K} K^{\prime} \simeq \mathrm{WD}\left(D \otimes_{K} K^{\prime}\right)^{\mathrm{ss}}=(r, N, V) \otimes_{K} K^{\prime},
$$

on a $\mathrm{WD}(D)^{\mathrm{ss}}=(r, N, V)$.

Remarque 2.6. On va voir plus loin que (cf. §4) si (ii) est vraie pour $K^{\prime}$, alors on peut toujours choisir une autre filtration admissible qui est stable par $\operatorname{Gal}\left(K^{\prime} / K\right)$. Donc en fait (ii) est vraie pour $K^{\prime}$ si et seulement si (ii) est vraie pour $K$.

\subsection{L'ordre des $\left(r_{i}, N_{i}, V_{i}\right)$}

On conserve les notations du paragraphe précédent. Supposons $K$ suffisamment gros pour pouvoir écrire

$$
(r, N, V)=\bigoplus_{i=1}^{s}\left(r_{i}, N_{i}, V_{i}\right)
$$

avec les $\left(r_{i}, N_{i}, V_{i}\right)$ absolument indécomposables de dimension $d_{i}$. Soit $\left(\varphi_{i}, N_{i}, \operatorname{Gal}\left(L^{\prime} / L\right), D_{i}\right)$ l'objet de $\mathrm{MOD}_{L^{\prime} / L}$ tel que $\mathrm{WD}\left(D_{i}\right)=\left(r_{i}, N_{i}, V_{i}\right)$, alors $D_{i}$ est absolument indécomposable dans $\mathrm{MOD}_{L^{\prime} / L}$. Posons $D_{i, 0}=\operatorname{Ker}\left(N_{i}: D_{i} \rightarrow D_{i}\right)$ et $\varphi_{i, 0}=\left.\varphi_{i}\right|_{D_{i, 0}}$, alors (notons que $r_{i}$ correspond au $L\left(b_{i}, \tau_{i}\right)$, cf. $\left.\S 2.2\right)$

$$
D_{i}=D_{i, 0} \oplus D_{i, 0}(1) \oplus \cdots \oplus D_{i, 0}\left(b_{i}-1\right),
$$

où $D_{i, 0}(n)=D_{i, 0}$ avec $\left.\varphi_{i}\right|_{D_{i, 0}(n)}=p^{n} \varphi_{i, 0}$, et où $\left.N_{i}\right|_{D_{i, 0}}=0$ et $N_{i}$ envoie $D_{i, 0}(n)$ dans $D_{i, 0}(n-1)$ par l'identité pour $n>0$. On dit que $D_{i}$ et $D_{j}$ sont de même type si

$$
\operatorname{Hom}_{\varphi, \operatorname{Gal}\left(L^{\prime} / L\right)}\left(D_{i}, D_{j}\right) \neq 0 .
$$

Remarquons que si $D_{i}$ et $D_{j}$ sont de même type, alors il existe un entier $l \in \mathbb{Z}$ tel que $D_{i, 0} \simeq D_{j, 0}(l)$ comme $\left(\varphi, \operatorname{Gal}\left(L^{\prime} / L\right)\right)$-modules.

On choisit un ordre des $\left(r_{i}, N_{i}, V_{i}\right)$ de telle sorte que (toujours possible) :

$$
\begin{aligned}
\{1, \ldots, s\} & =H_{1} \sqcup H_{2} \sqcup \cdots \sqcup H_{v} \\
& =\left\{1, \ldots, s_{1}\right\} \sqcup\left\{s_{1}+1, \ldots, s_{2}\right\} \sqcup \cdots \sqcup\left\{s_{r-1}+1, \ldots, s\right\}
\end{aligned}
$$

vérifiant les conditions suivantes :

- $k_{1}, k_{2} \in H_{i}$ si et seulement s'il existe $j_{1}, \ldots, j_{m}$ tels que $D_{k_{1}}$ et $D_{j_{1}}, D_{j_{i}}$ et $D_{j_{i+1}}$ $(1 \leq i \leq m-1), D_{j_{m}}$ et $D_{k_{2}}$ sont de même type;

- pour $i$ fixé, $k_{1}, k_{2} \in H_{i}, k_{1} \neq k_{2}$ et $D_{k_{1}, 0}(l) \simeq D_{k_{2}, 0}$ avec $l>0$ impliquent $k_{1}<k_{2}$;

- si $D_{k_{1}, 0} \simeq D_{k_{2}, 0}$, et $b_{k_{1}}<b_{k_{2}}$, alors $k_{1}<k_{2}$;

- Pour $i \neq j$, posons $M_{i}=\oplus_{k \in H_{i}} D_{k}, M_{j}=\oplus_{k \in H_{j}} D_{k}$. Si $t_{N}\left(M_{i}\right) / \operatorname{dim} M_{i}<$ $t_{N}\left(M_{j}\right) / \operatorname{dim} M_{j}$, alors $i<j$.

Proposition 2.7. Choisissons un ordre sur les $\left(r_{i}, N_{i}, V_{i}\right)$ comme précédemment, et soient $L\left(b_{i}, \tau_{i}\right)$ les représentations définies dans §2.2, alors la condition "ne précède pas" est vérifiée ([14], Definition 1.2.4). 
Démonstration. Supposons l'énoncé faux, et soit $i<j$ tel que $L\left(b_{i}, \tau_{i}\right)$ précède $L\left(b_{j}, \tau_{j}\right)$, ce qui implique $\tau_{i}(l)=\tau_{i}|\operatorname{det}|_{L}^{l} \simeq \tau_{j}$ pour un entier $l>0$ tel que $l+b_{j}>b_{i}$. Alors par la correspondance

$$
L\left(b_{i}, \tau_{i}\right) \leftrightarrow\left(r_{i}, N_{i}, V_{i}\right) \leftrightarrow D_{i},
$$

plus précisément,

$$
\tau_{i}|\operatorname{det}|_{L}^{l} \leftrightarrow D_{i, 0}\left(b_{i}-1-l\right), \quad \tau_{j} \leftrightarrow D_{j, 0}\left(b_{j}-1\right),
$$

on obtient

$$
D_{i, 0} \simeq D_{j, 0}\left(\left(l+b_{j}-b_{j}\right)\right),
$$

ce qui contredit $i<j$ puisque $l+b_{j}-b_{i}>0$.

\subsection{Module de Jacquet et un lemme d'Emerton}

On rappelle un lemme d'Emerton. On renvoie le lecteur à [8] ou [10] pour la définition d'une représentation localement analytique.

Soit $P$ un sous-groupe parabolique de $G$ avec $N$ son radical unipotent et $M$ son quotient de Levi. Dans [10], §3, est défini un foncteur "module de Jacquet" $J_{P}$ ayant la propriété suivante :

Proposition 2.8. Soient $\rho$ une $K$-représentation algébrique irréductible de $G$ et $\pi$ une $K$-représentation admissible lisse de $G$, alors il existe un isomorphisme canonique de $M$-représentations

$$
J_{P}\left(\rho \otimes_{K} \pi\right) \stackrel{\sim}{\rightarrow} \rho^{N} \otimes_{K}\left(r_{P}^{G} \pi\right) \delta_{P}^{1 / 2} .
$$

Démonstration. Voir [10], Proposition 4.3.6.

Si $N_{0}$ est un sous-groupe ouvert compact de $N$, on note $Z(M)$ le centre de $M$ et on pose

$$
Z(M)^{+}=\left\{z \in Z(M) \mid z N_{0} z^{-1} \subset N_{0}\right\} .
$$

Lemme 2.9. (Emerton) Avec les notations ci-dessus. Soient $V$ une représentation localement analytique de $G$ et $\chi: Z(M) \rightarrow K^{\times}$un caractère localement analytique de $Z(M)$. On considère les deux conditions :

(1) $V$ admet une norme invariante;

(2) $S i \operatorname{Hom}_{Z(M)}\left(\chi, J_{P}(V)\right) \neq 0$, alors

$$
\left|\chi(z) \delta_{P}^{-1}(z)\right|_{p} \leq 1, \quad \forall z \in Z(M)^{+} .
$$

Alors (1) entraîne (2).

Démonstration. Voir [9], Lemma 1.6 ou [10], Lemma 4.4.2.

Définition 2.10. On dit que $V$ satisfait à la condition d'Emerton si pour tout $P, N_{0}$ et $\chi$ comme ci-dessus la condition (2) du lemme 2.9 est vérifiée.

Remarque 2.11. Comme tout sous-groupe parabolique de $G$ est conjugué à un parabolique standard, on peut supposer que $P$ est un parabolique standard dans la définition 2.10. Aussi on peut supposer $N_{0}=N \cap \mathrm{M}_{d+1}\left(\mathbb{Z}_{p}\right)$, où $\mathrm{M}_{d+1}\left(\mathbb{Z}_{p}\right)$ est l'anneau des matrices carrées $(d+1) \times(d+1)$ à coefficients dans $\mathbb{Z}_{p}$. 


\subsection{Théorème principal}

Le résultat principal de cet article est le suivant.

Théorème 2.12. Supposons $K$ suffisamment gros tel que l'on a

$$
(r, N, V)=\bigoplus_{i=1}^{s}\left(r_{i}, N_{i}, V_{i}\right)
$$

avec les $r_{i}$ absolument indécomposables de dimension $d_{i}$. On considère les quatre conditions suivantes :

(i) $\rho \otimes \pi$ admet une norme invariante;

(ii) Il existe un objet $\left(\varphi, N, \operatorname{Gal}\left(L^{\prime} / L\right), D\right)$ dans $\mathrm{MOD}_{L^{\prime} / L}$ tel que :

$$
W D\left(\varphi, N, \operatorname{Gal}\left(L^{\prime} / L\right), D\right)^{\mathrm{ss}}=(r, N, V),
$$

et une filtration admissible $\left(\mathrm{Fil}^{i} D_{L^{\prime}, \sigma}\right)_{i, \sigma}$ stable par $\mathrm{Gal}\left(L^{\prime} / L\right) \times \operatorname{Gal}\left(K / \mathbb{Q}_{p}\right)$ sur $D_{L^{\prime}}$ telle que

$$
\mathrm{Fil}^{i} D_{L^{\prime}, \sigma} / \mathrm{Fil}^{i+1} D_{L^{\prime}, \sigma} \neq 0 \Leftrightarrow i \in\left\{i_{1, \sigma}, \ldots, i_{d+1, \sigma}\right\} .
$$

(iii) Avec l'ordre des $D_{i}$ comme en §2.4, les inégalités suivantes sont verifiées

$$
\begin{gathered}
{[K: L] \sum_{j=1}^{d_{1}} \sum_{\sigma} i_{j, \sigma} \leq t_{N}\left(D_{1}\right),} \\
\vdots \\
{[K: L] \sum_{j=1}^{d_{1}+\cdots+d_{s-1}} \sum_{\sigma} i_{j, \sigma} \leq \sum_{i=1}^{s-1} t_{N}\left(D_{i}\right),} \\
{[K: L] \sum_{j=1}^{d+1} \sum_{\sigma} i_{j, \sigma}=\sum_{i=1}^{s} t_{N}\left(D_{i}\right)=t_{N}(D) .}
\end{gathered}
$$

(iv) Le caractère central de $\rho \otimes \pi$ est unitaire et $V$ satisfait la condition d'Emerton. Alors, $(i) \Rightarrow(i i) \Leftrightarrow(i i i) \Leftrightarrow(i v)$.

Remarque 2.13. (1) L'implication " $(i i) \Rightarrow$ (iii)" découle de la définition d'admissibilité.

(2) L'implication " $(i) \Rightarrow(i v)$ " est une redite du lemme d'Emerton et [3], Proposition 5.1 .

Remarque 2.14. Si on pose $b=b_{1}+\cdots+b_{s}$,

$$
D_{1}^{\prime}=D_{1,0}, \ldots, D_{b_{1}}^{\prime}=D_{1,0}\left(b_{1}-1\right), \ldots, D_{b}^{\prime}=D_{s, 0}\left(b_{s}-1\right)
$$

et $d_{i}^{\prime}=\operatorname{rg}_{L_{0}^{\prime} \otimes_{\mathbb{Q} p} K} D_{i}^{\prime}$, alors pour toute permutation $\nu \in S_{b}$, on a

$$
[K: L] \sum_{j=1}^{d_{\nu(1)}^{\prime}} \sum_{\sigma} i_{j, \sigma} \leq t_{N}\left(D_{\nu(1)}^{\prime}\right)
$$




$$
\begin{gathered}
\vdots \\
{[K: L] \sum_{j=1}^{d_{\nu(1)}^{\prime}+\cdots+d_{\nu(b-1)}^{\prime}} \sum_{\sigma} i_{j, \sigma} \leq \sum_{i=1}^{b-1} t_{N}\left(D_{\nu(i)}^{\prime}\right),} \\
{[K: L] \sum_{j=1}^{d+1} \sum_{\sigma} i_{j, \sigma}=\sum_{i=1}^{b} t_{N}\left(D_{\nu(i)}^{\prime}\right)=t_{N}(D) .}
\end{gathered}
$$

Démonstration. Ceci résulte de l'ordre des $\left(r_{i}, N_{i}, V_{i}\right)$ (cf. §2.4).

Remarque 2.15. D'après le théorème, la conjecture 2.3 se "réduit" à (iv) $\Rightarrow$ (i) (supposons $K$ suffisamment gros). Il y a un résultat analogue dans le cas complexe (cf. [4], Theorem 4.4.6).

Corollaire 2.16. Avec les notations comme dans la conjecture 2.3, on a l'implication

$$
(i) \Rightarrow(i i)
$$

Démonstration. Ceci est une conséquence du théorème 2.12 et la proposition 2.5 .

Dans toute la suite de cet article, on suppose que $K$ est suffisamment gros au sens du théorème 2.12.

\section{Preuve de (iii) $\Leftrightarrow$ (iv)}

Dans ce chapitre, on prouve que (iii) entraîne (iv) (sous l'hypothèse que $K$ est suffisamment gros). Conservons les notations précédentes : $G=G L_{d+1}(L), d_{i}=b_{i} n_{i}$, $\alpha=\left(d_{1}, \ldots, d_{s}\right), \tau_{i}$ est une représentation cuspidale irréductible de $G_{d_{i}}, P=P_{\alpha}$ est le sous-groupe parabolique standard correspondant à la partition $\alpha, N_{\alpha}$ son radical unipotent, $M_{\alpha}$ son quotient de Levi, $\pi$ est l'unique modèle sur $K$ de la représentation

$$
\operatorname{Ind}_{P_{\alpha}}^{G} L\left(b_{1}, \tau_{1}\right) \otimes \cdots \otimes L\left(b_{s}, \tau_{s}\right) \otimes_{\overline{\mathbb{Q}}_{p}}|\operatorname{det}|_{L}^{-d / 2}
$$

$\rho$ est la représentation rationnelle de $G$ associées aux entiers $\left\{a_{j, \sigma}\right\}_{j, \sigma}$ et $\psi$ son plus haut poids.

\subsection{Le foncteur $r_{Q}^{G} \circ \operatorname{Ind}_{P}^{G}$}

On note $\tau=L\left(b_{1}, \tau_{1}\right) \otimes \cdots \otimes L\left(b_{s}, \tau_{s}\right)$. On fixe $Q=P_{\beta}$ un parabolique standard de $G$ où $\beta=\left(m_{1}, \ldots, m_{r}\right)$. Dans ce $\mathrm{n}^{\circ}$, on trouve une condition nécessaire pour qu'un caractère $\chi$ de $Z\left(M_{\beta}\right)$ soit tel que

$$
\operatorname{Hom}_{Z\left(M_{\beta}\right)}\left(\chi, r_{Q}^{G} \circ \operatorname{Ind}_{P}^{G}(\tau)\right) \neq 0 .
$$

D'abord on a besoin du lemme suivant : 
Lemme 3.1. Soient $l=k m$ et $\tau^{\prime}$ une représentation cuspidale irréductible de $G_{m}$. Si $\gamma=\left(l_{1}, \ldots, l_{n}\right)$ est une partition de $l$, alors $r_{P_{\gamma}}^{G_{l}}\left(L\left(k, \tau^{\prime}\right)\right) \neq 0$ si et seulement si $m \mid l_{i}$ pour tout $i$, et dans ce cas $r_{P_{\gamma}}^{G_{l}}\left(L\left(k, \tau^{\prime}\right)\right)$ est irréductible et égal à

$$
L\left(p_{n}, \tau^{\prime}|\operatorname{det}|^{p_{1}+\cdots+p_{n-1}}\right) \otimes \cdots \otimes L\left(p_{1}, \tau^{\prime}\right),
$$

où $p_{i}=l_{i} / m$. Son caractère central est égal à la restriction de

$$
\left(\chi\left(\tau^{\prime}\right)|\operatorname{det}|_{L}^{p_{1}+\cdots+p_{n-1}} \otimes \chi\left(\tau^{\prime}\right)|\operatorname{det}|_{L}^{k-1}\right) \otimes \cdots \otimes\left(\chi\left(\tau^{\prime}\right) \otimes \cdots \otimes \chi\left(\tau^{\prime}\right)|\operatorname{det}|_{L}^{p_{1}-1}\right)
$$

à $Z\left(M_{\gamma}\right)$, où $\chi\left(\tau^{\prime}\right)$ désigne le caractère central de $\tau^{\prime}$.

Démonstration. C'est une conséquence de la proposition 9.5 et la proposition 1.5 de [17.

$$
\begin{gathered}
\text { Posons } \gamma=(\underbrace{n_{1}, \ldots, n_{1}}_{b_{1} \text { fois }}, \underbrace{n_{2}, \ldots, n_{2}}_{b_{2} \text { fois }}, \ldots, \underbrace{n_{s}, \ldots, n_{s}}_{b_{s} \text { fois }}) \text { la partition de } d+1 \text {, et } \\
\quad \chi_{\gamma}=\left(\chi\left(\tau_{1}\right) \otimes \cdots \otimes \chi\left(\tau_{1}\right)|\operatorname{det}|_{L}^{b_{1}-1}\right) \otimes \cdots \otimes\left(\chi\left(\tau_{s}\right) \otimes \cdots \otimes \chi\left(\tau_{s}\right)|\operatorname{det}|_{L}^{b_{s}-1}\right)
\end{gathered}
$$

alors $\chi_{\gamma}$ est un caractère lisse de $Z\left(M_{\gamma}\right)$ dont le restriction à $Z\left(M_{\alpha}\right)$ est le caractère central de $\tau$. Posons $W \simeq S_{d+1}$ le groupe de Weyl de $G, P_{0}=P_{(1, \ldots, 1)}$ et

$$
W^{\alpha, \beta}=\left\{w \in W \mid w\left(M_{\alpha} \cap P_{0}\right) w^{-1} \subset P_{0}, w^{-1}\left(M_{\beta} \cap P_{0}\right) w \subset P_{0}\right\} .
$$

Si $w \in W^{\alpha, \beta}$ et on pose $\alpha^{\prime}=\alpha \cap w^{-1} \beta w$ (cf. [17], $\S 1.2$ ), alors $\alpha^{\prime} \leq \alpha$. Si de plus $\gamma \leq \alpha^{\prime}$, c'est-à-dire, $M_{\gamma} \subseteq M_{\alpha^{\prime}}$, on a $Z\left(M_{\alpha^{\prime}}\right) \subseteq Z\left(M_{\gamma}\right)$, via lequel on peut voir $\chi_{\gamma}$ comme un caractère de $M_{\alpha^{\prime}}$ et de même on voit $\chi_{\gamma}^{w^{-1}}$ comme un caractère de $M_{\beta^{\prime}}$, où par définition $\beta^{\prime}=w \alpha w^{-1} \cap \beta$ et $\chi^{w^{-1}}(z)=\chi\left(w^{-1} z w\right)$.

Proposition 3.2. (1) Si $P=Q$, on a $\operatorname{Hom}_{Z\left(M_{\beta}\right)}\left(\chi_{\gamma}, r_{P}^{G} \circ \operatorname{Ind}_{P}^{G}(\tau)\right) \neq 0$.

(2) $\operatorname{Hom}_{Z\left(M_{\beta}\right)}\left(\chi, r_{Q}^{G} \circ \operatorname{Ind}_{P}^{G}(\tau) \neq 0\right.$ si et seulement s'il existe une permutation $w \in$ $W^{\alpha, \beta}$ telle que

$$
\gamma \leq \alpha^{\prime}=\alpha \cap w^{-1} \beta w \text { et } \chi=\left.\chi_{\gamma}^{w^{-1}}\right|_{Z\left(M_{\beta}\right)} .
$$

Démonstration. (1) est un cas spécial de (2), et (2) se déduit de [17], Theorem 1.2 et Proposition 1.6, et du lemme 3.1.

Posons

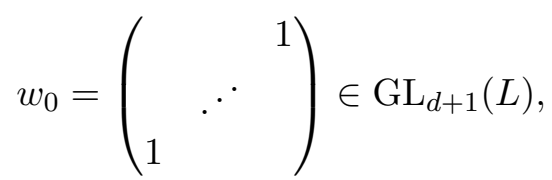

et $Q^{*}=w_{0} Q w_{0}^{-1}, N_{\beta}^{*}=w_{0} N_{\beta} w_{0}^{-1}, M_{\beta}^{*}=w_{0} M_{\beta} w_{0}^{-1}$.

Par la proposition 3.2 , on obtient

Corollaire 3.3. (1) Si $P=Q$, on a $\operatorname{Hom}_{Z\left(M_{\beta}^{*}\right)}\left(\left(\chi_{\gamma}\right)^{w_{0}} \psi \delta_{P^{*}}^{1 / 2}|\operatorname{det}|_{L}^{-d / 2}, J_{P^{*}}(\rho \otimes \pi)\right) \neq 0$;

(2) $\operatorname{Hom}_{Z\left(M_{\beta}^{*}\right)}\left(\chi, J_{Q^{*}}(\rho \otimes \pi)\right) \neq 0$ si et seulement s'il existe $w \in W^{\alpha, \beta}$ tel que

$$
\gamma \leq \alpha^{\prime}=\alpha \cap w^{-1} \beta w, \quad \text { et } \chi=\left(\left.\chi_{\gamma}^{w^{-1}}\right|_{Z\left(M_{\beta}^{*}\right)}\right)^{w_{0}} \psi \delta_{Q^{*}}^{1 / 2}|\operatorname{det}|_{L}^{-d / 2} .
$$


Démonstration. Ce corollaire se déduit de la proposition 3.2 et des faits suivants :

(a) d'après la proposition 2.8,

$$
J_{Q^{*}}(\rho \otimes \pi) \simeq \rho^{N_{\beta}^{*}} \otimes\left(r_{Q^{*}}^{G} \pi\right) \delta_{Q^{*}}^{1 / 2}=\rho^{N_{\beta}^{*}} \otimes\left(r_{Q^{*}}^{G} \circ \operatorname{Ind}_{P}^{G} \tau\right)|\operatorname{det}|_{L}^{-d / 2} \delta_{Q^{*}}^{1 / 2} ;
$$

(b) pour tout $Q$ parabolique standard, $\rho^{N^{*}}$ est une représentation algébrique irréductible de $M^{*}$ dont le caractère central est la restriction de $\psi$ à $Z\left(M^{*}\right)$;

(c) si $\sigma \in \operatorname{Rep} G$, alors $\operatorname{Hom}_{Z\left(M_{\beta}\right)}\left(\chi, r_{Q}^{G}(\sigma)\right) \neq 0$ si et seulement si $\operatorname{Hom}_{Z\left(M_{\beta}^{*}\right)}\left(\chi^{w_{0}}\right.$, $\left.r_{Q^{*}}^{G}(\sigma)\right) \neq 0$.

\subsection{La preuve}

$($ iii $) \Rightarrow($ iv $)$ : D'abord par [3], Proposition 5.1, le caractère central de $\rho \otimes \pi$ est unitaire.

Soit $\beta=\left(m_{1}, \ldots, m_{r}\right)$ une partition de $d+1$. Posons $Q=P_{\beta}, Q^{*}=w_{0} Q w_{0}^{-1}$, $N_{0}^{*}=N_{\beta}^{*} \cap \mathrm{M}_{d+1}\left(\mathbb{Z}_{p}\right) \subset N_{\beta}^{*}, Z\left(M_{\beta}^{*}\right)^{+}=\left\{z \in Z\left(M_{\beta}^{*}\right) \mid z N_{0}^{*} z^{-1} \subset N_{0}^{*}\right\}$. Soit $w \in W^{\alpha, \beta}$ tel que $\gamma \leq \alpha^{\prime}=\alpha \cap w^{-1} \beta w$. D'après le corollaire 3.3 et la remarque 2.11, il suffit de prouver

$$
\operatorname{val}_{p}\left(\chi_{\gamma}^{w^{-1} w_{0}} \psi \delta_{Q^{*}}^{-1 / 2}|\operatorname{det}|_{L}^{-d / 2}(z)\right) \geq 0, \quad \forall z \in Z\left(M_{\beta}^{*}\right)^{+} .
$$

On récrit $\chi_{\gamma}^{w^{-1}}$ sous la forme

$$
\chi_{1}^{\prime} \otimes \cdots \otimes \chi_{r}^{\prime}
$$

avec $\chi_{i}^{\prime}: Z\left(G_{m_{i}}\right) \rightarrow K^{\times}$. Soit $t_{1} \leq t_{2} \leq \cdots \leq t_{r}$ et on pose

$$
z=z\left(t_{1}, \ldots, t_{r}\right)=\operatorname{diag}\left(p^{t_{1}} \mathbb{1}_{m_{r}}, \ldots, p^{t_{r}} \mathbb{1}_{m_{1}}\right),
$$

où $\mathbb{1}_{m_{i}}$ désigne la matrice identité d'ordre $m_{i}$. Alors $z \in Z\left(M_{\beta}^{*}\right)^{+}$et on voit qu'il suffit de prouver $\left(^{*}\right)$ pour $z \in Z\left(M_{\beta}^{*}\right)^{+}$de cette forme.

On calcule $\operatorname{val}_{p}\left(\chi_{\gamma}^{w^{-1} w_{0}}(z)\right), \operatorname{val}_{p}(\psi(z)), \operatorname{val}_{p}\left(\delta_{Q^{*}}^{-1 / 2}(z)\right)$ et $|\operatorname{det}|_{L}^{-d / 2}$ respectivement.

(a) Trivialement on a

$$
\operatorname{val}_{p}\left(\chi_{\gamma}^{w^{-1} w_{0}}(z)\right)=\sum_{i=1}^{r} t_{s+1-i}\left(\operatorname{val}_{p}\left(\chi_{i}^{\prime}\left(p \mathbb{1}_{m_{i}}\right)\right)\right)
$$

et

$$
\operatorname{val}_{p}\left(|\operatorname{det}(z)|_{L}^{-d / 2}\right)=\frac{d}{2}\left[L: \mathbb{Q}_{p}\right] \sum_{i=1}^{r} m_{i} t_{r+1-i} .
$$

(b) Par la définition de $\rho$, on a

$$
\begin{aligned}
\operatorname{val}_{p}(\psi(z))= & t_{1}\left(\sum_{j=1}^{m_{r}} \sum_{\sigma} a_{j, \sigma}\right)+\cdots+t_{r}\left(\sum_{j=m_{r}+\cdots+m_{2}+1} \sum_{\sigma}^{d+1} a_{j, \sigma}\right) \\
= & \left.-t_{1} \sum_{j=1}^{m_{r}} \sum_{\sigma} i_{d+2-j, \sigma}-\cdots-t_{r} \sum_{j=m_{r}+\cdots+m_{2}+1}^{\sum_{m_{1}(2} \sum_{j>1} i_{d+2-j, \sigma}} i_{j}+m_{1}-1\right) \\
& -\left[L: \mathbb{Q}_{p}\right] t_{1} \frac{m_{r}\left(m_{r}-1\right)}{2}-\cdots-t_{r}\left[L: \mathbb{Q}_{p}\right] \frac{\sum_{m_{1}+\cdots+m_{i}}}{=}-\sum_{i=1}^{r}\left(t_{r+1-i} \sum_{j=m_{1}+\cdots+m_{i-1}+1} i_{j, \sigma}\right) \\
& -\frac{1}{2}\left[L: \mathbb{Q}_{p}\right]\left(\sum_{i=1}^{r} t_{r+1-i} m_{i}\left(2 \sum_{j>i} m_{j}+m_{i}-1\right)\right) .
\end{aligned}
$$


(c) Par [16], chapitre I.2.7, exemple (c), on a

$$
\begin{aligned}
\operatorname{val}_{p}\left(\delta_{Q^{*}}^{-1 / 2}(z)\right) & =\frac{1}{2} \operatorname{val}_{p}\left(\delta_{Q}^{-1}\left(w_{0} z w_{0}^{-1}\right)\right) \\
& =-\frac{1}{2}\left[L: \mathbb{Q}_{p}\right] \sum_{i=1}^{r}\left(\sum_{j<i} m_{j}-\sum_{j>i} m_{j}\right) m_{i} t_{r+1-i} .
\end{aligned}
$$

Un calcul rapide implique que

$$
\begin{aligned}
& \operatorname{val}_{p}\left(\left.\chi_{\gamma}^{w^{-1} w_{0}} \psi \delta_{Q^{*}}^{-1 / 2} \operatorname{det}\right|_{L} ^{-d / 2}(z) \mid\right) \\
= & \sum_{i=1}^{r} t_{r+1-i} \operatorname{val}_{p}\left(\chi_{i}^{\prime}\left(p \mathbb{1}_{m_{i}}\right)\right)-\sum_{i=1}^{r}\left(t_{r+i-1} \sum_{j=m_{1}+\cdots+m_{i-1}+1}^{m_{1}+\cdots+m_{i}} \sum_{\sigma} i_{j, \sigma}\right) .
\end{aligned}
$$

Notons que, par la correspondance de Langlands locale unitaire (non modifiée), le caractère central de $L\left(b_{i}, \tau_{i}\right)$ est $\operatorname{det}\left(r_{i}, N_{i}, V_{i}\right) \circ \mathrm{rec}^{-1}$, on a donc pour tout $1 \leq i \leq s$ (cf. [3], Proposition 5.1)

$$
\chi\left(\tau_{i}\right)|\operatorname{det}|_{L}^{j}\left(p \mathbb{1}_{m_{i}}\right)=\frac{1}{[K: L]} t_{N}\left(D_{i, 0}\left(b_{i}-1-j\right)\right), \quad j=0, \ldots, b_{i}-1 ;
$$

mais, par définition, $\chi_{i}^{\prime}$ est un produit de tels caractères, on en déduit les inégalités ${ }^{*}$ ) de la remarque 2.14 et du fait que $t_{1} \leq t_{2} \leq \cdots \leq t_{r}$.

(iv) $\Rightarrow$ (iii) : Immédiat à partir du calcul ci-dessus.

Remarque 3.4. Cette preuve est une généralisation de [9], Lemma 2.1.

\section{$4 \quad$ Preuve de (iii) $\Rightarrow$ (ii)}

Supposons que (iii) est vrai, on va prouver (ii) dans ce chapitre. Posons

$$
\left(\varphi_{i}, N_{i}, \operatorname{Gal}\left(L^{\prime} / L\right), D_{i}\right)=\operatorname{MOD}\left(r_{i}, N_{i}, V_{i}\right) .
$$

Convention : comme les $L_{0}^{\prime} \otimes_{\mathbb{Q}_{p}} K$-modules $D^{\prime}$ qu'on va traiter sont tous objets de $\mathrm{MOD}_{L^{\prime} / L}$ libres de rang égal à $\operatorname{dim}_{K}\left(\mathrm{WD}\left(D^{\prime}\right)\right)$, on pose $\operatorname{dim} D^{\prime}=\operatorname{rg}_{L_{0}^{\prime} \otimes_{\mathbb{Q}_{p}} K} D^{\prime}$.

\subsection{Construction de $\left(\varphi, N, \operatorname{Gal}\left(L^{\prime} / L\right), D\right)$}

Dans ce paragraphe, on construit un objet $\left(\varphi, N, \operatorname{Gal}\left(L^{\prime} / L\right), D\right)$ de $\mathrm{MOD}_{L^{\prime} / L}$ tel que

$$
\mathrm{WD}\left(\varphi, N, \operatorname{Gal}\left(L^{\prime} / L\right), D\right)^{\mathrm{ss}}=(r, N, V) .
$$

D'abord, on regarde quelques exemples.

Exemples 4.1. On considère le cas $L^{\prime}=L=\mathbb{Q}_{p}$ (donc $\operatorname{Gal}\left(L^{\prime} / L\right)=\{1\}$ ). Définissons $\left(D_{0}, \varphi_{0}, N_{0}\right)$ par :

$$
D_{0}=K e, \varphi_{0}(e)=e, N_{0}(e)=0 .
$$

(1) (a) Soit $D=D_{1} \oplus D_{2}=D_{0} \oplus\left(D_{0} \oplus D_{0}(1)\right)$, c'est-à-dire,

$$
D=K^{3}=K e_{1} \oplus K e_{2} \oplus K e_{3}
$$


avec

$$
\varphi\left(e_{1}\right)=e_{1}, \quad \varphi\left(e_{2}\right)=e_{2}, \quad \varphi\left(e_{3}\right)=p e_{3}
$$

et

$$
N\left(e_{1}\right)=0, \quad N\left(e_{2}\right)=0, \quad N\left(e_{3}\right)=e_{2} .
$$

Alors il existe un triplet $\left(i_{1}, i_{2}, i_{3}\right)$ tel que

$$
i_{1}<i_{2}<i_{3}, \quad i_{1}+i_{2}+i_{3}=t_{N}(D)=1,
$$

et tel qu'il n'existe aucune filtration admissible dont les poids de Hodge-Tate (i.e. les entiers $i$ tels que $\mathrm{Fil}^{-i} D / \mathrm{Fil}^{-i+1} D \neq 0$ avec multiplicité) soient $\left\{-i_{1},-i_{2},-i_{3}\right\}$. En fait, on peut choisir un triplet $\left(i_{1}, i_{2}, i_{3}\right)$ tel que

$$
i_{1}<i_{2}<i_{3}, \quad i_{1}+i_{2}+i_{3}=t_{N}(D)=1, \text { et } i_{2}>0 .
$$

Soit $\left(\mathrm{Fil}^{i} D\right)_{i}$ une filtration quelconque dont les poids de Hodge-Tate sont $\left\{-i_{1},-i_{2},-i_{3}\right\}$. Alors $\operatorname{dimFil}{ }^{i_{2}} D=2$ et si on pose $D^{\prime}=\mathrm{Fil}^{i_{2}} D \cap\left(K e_{1} \oplus K e_{2}\right)$ qui est un sous-objet de $D$, on a

$$
\begin{cases}t_{H}\left(D^{\prime}\right)=i_{2}+i_{3}>0=t_{N}\left(D^{\prime}\right) & \text { si Fil }{ }^{i_{2}} D=K e_{1}+K e_{2} \\ t_{H}\left(D^{\prime}\right) \geq i_{2}>0=t_{N}\left(D^{\prime}\right) & \text { sinon. }\end{cases}
$$

La situation sera améliorée si on modifie $\varphi$ par

$$
\varphi^{*}\left(e_{1}\right)=e_{1}+e_{2}, \quad \varphi^{*}\left(e_{2}\right)=e_{2}, \quad \varphi^{*}\left(e_{3}\right)=p e_{3} .
$$

On vérifie que $p \varphi^{*} N=N \varphi^{*}$, et tout sous-objet $D^{\prime}$ non trivial de $D$ est de la forme :

$$
K e_{2} ; \quad K e_{i} \oplus K e_{j},(i, j)=(1,2) \text { ou }(2,3) .
$$

On peut construire une filtration telle que

$$
t_{H}\left(K e_{i}\right)=i_{1}, \quad t_{H}\left(K e_{i} \oplus K e_{j}\right)=i_{1}+i_{2}
$$

pour $i \neq j$ (cf. lemme 4.8 ci-après), et la condition $i_{1}<i_{2}<i_{3}$ entraîne que cette filtration est admissible.

(b) La même preuve pour $D=\left(D_{0} \oplus D_{0}(1)\right) \oplus D_{0}(1)$.

(2) Soit $D=D_{1} \oplus D_{2}=\left(D_{0} \oplus D_{0}(1)\right) \oplus\left(D_{0}(1) \oplus D_{0}(2)\right)$, c'est-à-dire,

$$
\begin{gathered}
D=K e_{1} \oplus K e_{2} \oplus K e_{3} \oplus K e_{4}, \\
\varphi\left(e_{1}\right)=e_{1}, \quad \varphi\left(e_{2}\right)=p e_{2}, \quad \varphi\left(e_{3}\right)=p e_{3}, \quad \varphi\left(e_{4}\right)=p^{2} e_{4} \\
N\left(e_{1}\right)=0, \quad N\left(e_{2}\right)=e_{1}, \quad N\left(e_{3}\right)=0, \quad N\left(e_{4}\right)=e_{3} .
\end{gathered}
$$

On peut énumérer tous les sous-objets non triviaux de $D$ stables par $\varphi, N$ :

$$
\begin{gathered}
K e_{i}, i=1,3 ; \quad K e_{i} \oplus K e_{j},(i, j)=(1,2),(1,3),(3,4) \\
K e_{i} \oplus K e_{j} \oplus K e_{k},(i, j, k)=(1,2,3),(1,3,4) \\
K e_{1} \oplus K v, \quad \text { avec } v=a e_{2}+b e_{3}, a, b \neq 0 .
\end{gathered}
$$


Soient $\left\{i_{j}\right\}_{1 \leq j \leq 4}$ des entiers tels que

$$
\sum_{j=1}^{4} i_{j}=4, \quad i_{1}<i_{2}<i_{3}<i_{4} .
$$

On peut construire une filtration $\left(\mathrm{Fil}^{i} D\right)_{i}$ sur $D$ telle que (cf. lemme 4.8)

$$
\mathrm{Fil}^{i} D / \mathrm{Fil}^{i+1} D \neq 0 \Leftrightarrow i \in\left\{i_{1}, i_{2}, i_{3}, i_{4}\right\}
$$

et telle que

$$
t_{H}\left(K e_{i}\right)=i_{1}, \quad t_{H}\left(K e_{i} \oplus K e_{j}\right)=i_{1}+i_{2}, \quad t_{H}\left(K e_{i} \oplus K e_{j} \oplus K e_{k}\right)=i_{1}+i_{2}+i_{3},
$$

pour tous $i, j, k$ distincts. En particulier, si $D^{\prime}=K e_{1} \oplus K v$ avec $v=a e_{2}+b e_{3}, a, b \neq 0$, on a $t_{H}\left(D^{\prime}\right) \leq i_{1}+i_{3}$. L'hypothèse sur $\left\{i_{j}\right\}_{1 \leq j \leq 4}$ implique $i_{1}+i_{3} \leq 1$ et on en déduit que la filtration est admissible.

Notons qu'on peut aussi modifier $\varphi$ comme en (1) :

$$
\varphi^{*}\left(e_{2}\right)=p e_{2}+p e_{3}, \quad \varphi^{*}\left(e_{i}\right)=\varphi e_{i} \text { pour } i \neq 2 .
$$

On vérifie que $p \varphi^{*} N=N \varphi^{*}$ et

$$
W D\left(\varphi, N, \operatorname{Gal}\left(L^{\prime} / L\right), D\right)^{\mathrm{ss}}=W D\left(\varphi^{*}, N, \operatorname{Gal}\left(L^{\prime} / L\right), D\right)^{\mathrm{ss}},
$$

et que la filtration définie ci-dessus est encore admissible.

(3) Soit $D=D_{1} \oplus D_{2}=\left(D_{0} \oplus D_{0}(1) \oplus D_{0}(2)\right) \oplus D_{0}(1)$. De façon plus précise,

$$
\begin{gathered}
D=K e_{1} \oplus K e_{2} \oplus K e_{3} \oplus K e_{4} ; \\
\varphi\left(e_{1}\right)=e_{1}, \quad \varphi\left(e_{2}\right)=p e_{2}, \quad \varphi\left(e_{3}\right)=p^{2} e_{3}, \quad \varphi\left(e_{4}\right)=p e_{4} \\
N\left(e_{1}\right)=0, \quad N\left(e_{2}\right)=e_{1}, \quad N\left(e_{3}\right)=e_{2}, \quad N\left(e_{4}\right)=0 .
\end{gathered}
$$

On peut vérifier que si $\varphi^{*}$ est un endomorphisme de $D$ tel que $p \varphi^{*} N=N \varphi^{*}$ et tel que

$$
W D\left(\varphi^{*}, N, \operatorname{Gal}\left(L^{\prime} / L\right), D\right)^{\mathrm{ss}}=W D\left(\varphi, N, \operatorname{Gal}\left(L^{\prime} / L\right), D\right)^{\mathrm{ss}},
$$

alors $\varphi^{*}=\varphi$.

Remarque 4.2. (1) L'exemple dans (1) nous dit que l'on doit modifier $\varphi$ dans certains cas. (Notons que le triplet $\left(i_{1}, i_{2}, i_{3}\right)$ vérifie la condition (iii) du théorème 2.12.)

(2) L'exemple (2) signale qu'il n'est pas toujours nécessaire de modifier $\varphi$.

(3) L'exemple (3) nous dit qu'il n'est pas toujours possible de modifier $\varphi$.

Lemme 4.3. On fixe un $H_{i}$, et soit $k_{1} \in H_{i}, k_{2} \in H_{i}$ tels que $k_{1}<k_{2}$. On peut écrire

$$
\begin{gathered}
D_{k_{1}}=D_{0} \oplus D_{0}(1) \oplus \cdots \oplus D_{0}\left(r_{1}\right), \\
D_{k_{2}}=D_{0}(l) \oplus D_{0}(l+1) \oplus \cdots \oplus D_{0}\left(l+r_{2}\right),
\end{gathered}
$$

avec $D_{0}=\operatorname{ker}\left(N: D_{k_{1}} \rightarrow D_{k_{1}}\right)$ absolument irréductible et $l \geq 0$. Alors 
(1) Pour que $\operatorname{Hom}_{\mathrm{MOD}_{L^{\prime} / L}}\left(D_{k_{1}}, D_{k_{2}}\right) \neq 0$ il faut et il suffit que $l \leq r_{1} \leq l+r_{2}$. S'il en est ainsi, $\operatorname{Hom}_{\mathrm{MOD}_{L^{\prime} / L}}\left(D_{k_{1}}, D_{k_{2}}\right)$ est un $K$-espace vectoriel de dimension 1.

(2) Supposons $\operatorname{Hom}_{\mathrm{MOD}_{L^{\prime} / L}}\left(D_{k_{1}}, D_{k_{2}}\right) \neq 0$ et soit $\alpha: D_{k_{1}} \rightarrow D_{k_{2}}$ un tel morphisme non nul. On modifie le Frobenius sur $D_{k_{1}} \oplus D_{k_{2}}$ en posant $\varphi: D_{k_{1}} \oplus D_{k_{2}} \rightarrow D_{k_{1}} \oplus D_{k_{2}}$,

$$
\varphi\left(e_{1}\right)=\varphi_{k_{1}}\left(e_{1}\right)+\varphi_{k_{2}}\left(\alpha\left(e_{1}\right)\right), \quad \varphi\left(e_{2}\right)=\varphi_{k_{2}}\left(e_{2}\right),
$$

où $\varphi_{k_{i}}$ est le Frobenius sur $D_{k_{i}}$. Alors $p \varphi N=N \varphi$ où $N=N_{k_{1}} \oplus N_{k_{2}}: D_{k_{1}} \oplus D_{k_{2}} \rightarrow$ $D_{k_{1}} \oplus D_{k_{2}}$. De plus, on a

$$
\left(\varphi, N, \operatorname{Gal}\left(L^{\prime} / L\right), D_{k_{1}} \oplus D_{k_{2}}\right) \in \operatorname{MOD}_{L^{\prime} / L}
$$

et

$\mathrm{WD}\left(\varphi, N, \operatorname{Gal}\left(L^{\prime} / L\right), D_{k_{1}} \oplus D_{k_{2}}\right)^{\mathrm{ss}}=\mathrm{WD}\left(\varphi_{k_{1}} \oplus \varphi_{k_{2}}, N, \operatorname{Gal}\left(L^{\prime} / L\right), D_{k_{1}} \oplus D_{k_{2}}\right)^{\mathrm{ss}}$

(3) Tout sous-objet de $\left(\varphi, N, \operatorname{Gal}\left(L^{\prime} / L\right), D_{k_{1}} \oplus D_{k_{2}}\right)$ est, à isomorphisme près, de la forme :

$$
\left(D_{0} \oplus \cdots \oplus D_{0}\left(n_{1}\right)\right) \oplus\left(D_{0}(l) \oplus \cdots \oplus D_{0}\left(l+n_{2}\right)\right)
$$

avec $0 \leq n_{1} \leq r_{1}, 0 \leq n_{2} \leq r_{2}$ vérifiant $n_{1} \leq l$ ou $l \leq n_{1} \leq l+n_{2}$.

Démonstration. Immédiat.

Motivé par les exemples 4.1 et le lemme 4.3 ci-dessus, on définit l'objet $\left(\varphi, N, \operatorname{Gal}\left(L^{\prime} / L\right), D\right)$ de la manière suivante :

- en tant que $L_{0}^{\prime} \otimes_{\mathbb{Q}_{p}} K$-module, $D=\oplus_{i=1}^{s} D_{i}$;

- on définit $N=\oplus_{i=1}^{s} N_{i}$ sur $D$;

- on définit l'action de $\operatorname{Gal}\left(L^{\prime} / L\right)$ naturellement;

- pour tout $1 \leq k_{1}<k_{2} \leq s$ :

(a) si $k_{1}, k_{2} \in H_{i}$ pour un $i$, on peut écrire

$$
D_{k_{1}}=D_{0} \oplus \cdots \oplus D_{0}\left(r_{1}\right), \quad D_{k_{2}}=D_{0}(l) \oplus \cdots \oplus D_{0}\left(l+r_{2}\right) ;
$$

avec $r_{1}, r_{2}, l \geq 0$. Si de plus $l=0$ ou $r_{1}=l+r_{2}$, et $k_{2}$ est le plus petit entier de $H_{i}$ ayant l'une de ces propriétés, on pose ( $\operatorname{sur} D_{k_{1}} \oplus D_{k_{2}}$ )

$$
\varphi\left(e_{1}\right)=\varphi_{k_{1}}\left(e_{1}\right)+\varphi_{k_{2}}\left(\alpha\left(e_{1}\right)\right) \text { si } e_{1} \in D_{k_{1}}, \quad \varphi\left(e_{2}\right)=e_{2} \text { si } e_{2} \in D_{k_{2}}
$$

où $\alpha \neq 0$ est un élément fixé de $\operatorname{Hom}_{\mathrm{MOD}_{L^{\prime} / L}}\left(D_{k_{1}}, D_{k_{2}}\right)$.

(b) sinon, on pose $\varphi=\varphi_{k_{1}} \oplus \varphi_{k_{2}}$ sur $D_{k_{1}} \oplus D_{k_{2}}$.

On déduit de la définition et du lemme 4.3 que $\left(\varphi, N, \operatorname{Gal}\left(L^{\prime} / L\right), D\right) \in \mathrm{MOD}_{L^{\prime} / L}$ et

$$
\mathrm{WD}\left(\varphi, N, \operatorname{Gal}\left(L^{\prime} / L\right), D\right)^{\mathrm{ss}}=(r, N, V) .
$$




\section{2 $\quad$ Structure de $D$}

On conserve les notations du paragraphe précédent. Dans ce paragraphe, on étudie la structure de $D$ qui sera utilisée dans la suite.

D'abord, on suppose $D=D_{H_{i}}$ pour un $i$ de sorte qu'on peut écrire

$$
\begin{aligned}
D & =D_{1} \oplus \cdots \oplus D_{s} \\
& =\left(D_{0} \oplus D_{0}(1) \oplus \cdots \oplus D_{0}\left(b_{1}-1\right)\right) \oplus \cdots \oplus\left(D_{0}\left(l_{s}\right) \oplus \cdots \oplus D_{0}\left(l_{s}+b_{s}-1\right)\right)
\end{aligned}
$$

avec $l_{i} \geq 0$ et $D_{0}$ absolument irréductible de dimension $h$.

On considère pour l'instant $\left(\left.\varphi\right|_{D_{1}},\left.N\right|_{D_{1}}, \operatorname{Gal}\left(L^{\prime} / L\right), D_{1}\right) \in \mathrm{MOD}_{L^{\prime} / L}$. Par hypothèse, $\left(\left.\varphi\right|_{D_{0}}, \operatorname{Gal}\left(L^{\prime} / L\right), D_{0}\right)$ est un objet absolument irréductible dans $\mathrm{MOD}_{L^{\prime} / L}$, donc d'après la remarque 2.1. $\left.\varphi^{f^{\prime}}\right|_{D_{0}}=a$ est scalaire avec $a \in K^{\times}$. Notons $\varphi^{\prime}=\varphi^{f^{\prime}}$ et $q^{\prime}=p^{f^{\prime}}$.

On récrit $D$ sous la forme

$$
\begin{aligned}
D & =D_{=0} \oplus \cdots \oplus D_{=n} \\
& =\left(\oplus D_{0}\right) \oplus\left(\oplus D_{0}(1)\right) \oplus \cdots \oplus\left(\oplus D_{0}(n)\right),
\end{aligned}
$$

où $n=\max _{2 \leq i \leq s}\left\{r_{1}, l_{i}+b_{i}-1\right\}$. Alors par définition de $\varphi$

$$
D_{=j}=\left\{v \in D \mid\left(\varphi^{\prime}-q^{\prime j} a\right)^{k} v=0, k \gg 0\right\} .
$$

Définition 4.4. (1) Un sous-objet $D^{\prime}$ de $D$ est dit bon s'il est de la forme

$$
\begin{aligned}
D^{\prime} & =D_{1}^{\prime} \oplus \cdots \oplus D_{s}^{\prime} \\
& =\left(D_{0} \oplus D_{0}(1) \oplus \cdots \oplus D_{0}\left(b_{1}^{\prime}\right)\right) \oplus \cdots \oplus\left(D_{0}\left(l_{s}\right) \oplus \cdots \oplus D_{0}\left(l_{s}+b_{s}^{\prime}\right)\right)
\end{aligned}
$$

avec $-1 \leq b_{i}^{\prime} \leq b_{i}-1$, où $b_{i}^{\prime}=-1$ désigne que $D^{\prime} \cap D_{i}=0$.

(2) On appelle drapeau de $D$ une suite de sous- $L_{0} \otimes_{\mathbb{Q}_{p}} K$-modules libres $\left\{E_{i}\right\}_{1 \leq i \leq m}$ de $D$ tels que

$$
0 \subsetneq E_{1} \subsetneq \cdots \subsetneq E_{m} \subsetneq D
$$

soit

$$
0 \subsetneq E_{1}^{\prime} \subsetneq \cdots \subsetneq E_{m^{\prime}}^{\prime} \subsetneq D
$$

un autre drapeau de $D$, il est dite un sous-drapeau s'il existe $1 \leq j(i) \leq m^{\prime}$ tel que $E_{i}=E_{j(i)}^{\prime}$ pour tout $1 \leq i \leq m$. Soit $\sigma: L \hookrightarrow K$ un plongement, on définit un drapeau de $D_{L^{\prime}, \sigma}$ de manière analogue.

(3) Un drapeau

$$
\Delta: 0 \subsetneq E_{1} \subsetneq \cdots \subsetneq E_{m} \subsetneq D
$$

de $D$ est dit bon si tous les $E_{i}$ sont bons.

Remarque 4.5. (1) L'ensemble des bons sous-objets de $D$ est de cardinal fini.

(2) Si $E_{1}$ et $E_{2}$ sont deux bons sous-objets de $D$, alors $E_{1}+E_{2}, E_{1} \cap E_{2}, \operatorname{ker}\left(\left.N\right|_{E_{1}}\right)$ et $N\left(E_{1}\right)$ sont bons aussi. De plus, pour tout $j, \operatorname{ker}\left(\left.\left(\varphi^{\prime}-q^{\prime j} a\right)\right|_{E_{1}}\right)$ et $\left(\varphi^{\prime}-q^{\prime j} a\right)\left(E_{1}\right)$ sont bons.

Si on définit

$$
D_{=j, i}=\left\{v \in D_{=j} \mid\left(\varphi^{\prime}-q^{\prime j} a\right)^{i} v=0\right\}
$$


alors on obtient un bon drapeau de $D_{=j}$ :

$$
D_{=j, 1} \subset D_{=j, 2} \subset \cdots .
$$

Posons $D_{<j}=\oplus_{i<j} D_{=i}$ et $D_{\leq j}=\oplus_{i \leq j} D_{=i}$.

Dans le cas général $D=\oplus_{i} D_{H_{i}}$, on dit qu'un sous-objet $D^{\prime}$ est bon s'il est somme directe de bons sous-objets de $D_{H_{i}}$.

\subsection{Définition de la filtration sur $D$}

On conserve les notations du paragraphe précédent. Soit $\mathrm{Fil}_{\sigma}=\left(\mathrm{Fil}^{i} D_{L^{\prime}, \sigma}\right)_{i}$ une filtration de $D_{L^{\prime}, \sigma}$ stable par $\operatorname{Gal}\left(L^{\prime} / L\right)$. On note $I\left(\mathrm{Fil}_{\sigma}\right)$ l'ensemble des entiers $i$ tels que

$$
\mathrm{Fil}^{i} D_{L^{\prime}, \sigma} / \mathrm{Fil}^{i+1} D_{L^{\prime}, \sigma} \neq 0
$$

avec multiplicité égale à $\operatorname{dim}_{K} \mathrm{Fil}^{i} D_{L^{\prime}, \sigma} / \mathrm{Fil}^{i+1} D_{L^{\prime}, \sigma}$ (i.e. les opposés des poids de HodgeTate).

Remarque 4.6. Si $D_{1} \subset D_{2}$ sont deux sous- $L^{\prime} \otimes_{L, \sigma} K$-modules de $D_{L^{\prime}, \sigma}$. Alors $I\left(\mathrm{Fil}_{\sigma}, D_{1}\right)$ $\subset I\left(\mathrm{Fil}_{\sigma}, D_{2}\right)$.

Définition 4.7. Soit

$$
\Delta_{\sigma}: 0=E_{0, \sigma} \subsetneq E_{1, \sigma} \subsetneq \cdots \subsetneq E_{m+1, \sigma}=D_{L^{\prime}, \sigma}
$$

un drapeau de $D_{L^{\prime}, \sigma}$ par des sous- $L^{\prime} \otimes_{L, \sigma} K$-modules libres, et $\mathrm{Fil}_{\sigma}$ une filtration sur $D_{L^{\prime}, \sigma}$ avec $I\left(\right.$ Fil $\left._{\sigma}\right)=\left\{i_{1, \sigma}<i_{2, \sigma}<\cdots<i_{d+1, \sigma}\right\}$. On dit que Fil $\sigma$ est transverse à $\Delta_{\sigma}$, si pour tout $k$,

$$
I\left(\mathrm{Fil}_{\sigma}, E_{k, \sigma}\right)=\left\{i_{1, \sigma}, \ldots, i_{\operatorname{dim}_{K} E_{k, \sigma}, \sigma}\right\} .
$$

Lemme 4.8. Soient $\Delta=\left(\Delta_{\sigma}\right)_{\sigma}$ un drapeau de $D$, et pour tout $\sigma: L \hookrightarrow K$ des entiers $\left\{i_{j, \sigma}\right\}_{1 \leq j \leq d+1}$ tels que $i_{1, \sigma}<\cdots<i_{d+1, \sigma}$. Alors il existe une filtration Fil $=\left(\mathrm{Fil}_{\sigma}\right)_{\sigma}$ sur $D$ stable par $\operatorname{Gal}\left(L^{\prime} / L\right)$ telle que pour tout $\sigma, \mathrm{Fil}_{\sigma}$ est transverse $\grave{a} \Delta_{\sigma}$ et

$$
I\left(\mathrm{Fil}_{\sigma}\right)=\left\{i_{1, \sigma}, \ldots, i_{d+1, \sigma}\right\} .
$$

De plus, si $K_{1} \subset K$ est un sous-corps de cardinal infini, alors on peut prendre Fil $_{\sigma}$ telle qu'elle soit définie sur $K_{1}$.

Démonstration. Voir [3, Proposition 3.2.

Remarque 4.9. De plus, si on se donne $\Delta_{1}, \ldots, \Delta_{n}$ des drapeaux de $D$ et des entiers $\left\{i_{1, \sigma}<\cdots<i_{d+1, \sigma}\right\}$, on obtient encore par la preuve du lemme 4.8 qu'il existe une filtration Fil telle que l'énoncé du lemme 4.8 soit vrai pour tout $\Delta_{i}$.

On fixe une filtration Fil $=\left(\mathrm{Fil}_{\sigma}\right)_{\sigma}$ sur $D$ qui est transverse à tous les bons drapeaux (c'est un ensemble fini). En particulier, comme tout bon sous-objet $D^{\prime}$ de $D$ appartient à un tel drapeau, on a (cf. la preuve de [3], Proposition 5.1)

$$
t_{H}\left(D_{L^{\prime}}^{\prime}\right)=[K: L] \sum_{j=1}^{\operatorname{dim} D^{\prime}} \sum_{\sigma} i_{j, \sigma} .
$$


Corollaire 4.10. Avec les notations précédentes. Soient $\left\{E_{i}\right\}_{0 \leq i \leq m+1}$ des bons sousobjets de $D$ tels que $E_{0}=0, E_{m+1}=D$ et $\operatorname{dim} E_{i-1}<\operatorname{dim} E_{i}$, et $D^{\prime}$ un sous-objet quelconque de D. Posons

$$
a_{i}=\operatorname{dim} E_{i}-\operatorname{dim} E_{i-1}, \quad c_{i}=\operatorname{dim}\left(E_{i} \cap D^{\prime}\right)-\operatorname{dim}\left(E_{i-1} \cap D^{\prime}\right),
$$

et

$$
\Omega=\left\{j \in \mathbb{N} \mid \text { il existe } l \text { tel que } \sum_{i=0}^{l} a_{i}-c_{l}+1 \leq j \leq \sum_{i=0}^{l} a_{i}\right\}
$$

Alors

$$
t_{H}\left(D_{L^{\prime}}^{\prime}\right) \leq[K: L] \sum_{j \in \Omega} \sum_{\sigma} i_{j, \sigma}
$$

Démonstration. Cela découle de la définition [4.7, de la remarque 4.6] et du choix de Fil.

\subsection{La preuve : cas spécial}

Dans ce paragraphe, on va prouver l'implication "(ii) $\Rightarrow$ (iii)" du théorème 2.12 dans le cas spécial $D=D_{H_{i}}$. Rappelons qu'on a défini un certain objet $\left(\varphi, N, \operatorname{Gal}\left(L^{\prime} / L\right), D\right)$ (cf. §4.1) de $\mathrm{MOD}_{L^{\prime} / L}$ tel que

$$
\mathrm{WD}\left(\varphi, N, \operatorname{Gal}\left(L^{\prime} / L\right), D\right)^{\mathrm{ss}}=(r, N, V)
$$

et on a fixé une filtration Fil $=\left(\mathrm{Fil}^{i} D_{L^{\prime}, \sigma}\right)_{i, \sigma}(\mathrm{cf}$. $\$ 4.3)$ sur $D_{L^{\prime}}$ telle que

$$
\mathrm{Fil}^{i} D_{L^{\prime}, \sigma} / \mathrm{Fil}^{i+1} D_{L^{\prime}, \sigma} \neq 0 \Leftrightarrow i \in\left\{i_{1, \sigma}, \ldots, i_{d+1, \sigma}\right\} .
$$

Donc il suffit de prouver

Théorème 4.11. La filtration Fil sur $D_{L^{\prime}}$ est admissible. Autrement dit, si $D^{\prime}$ est un sous-objet de D, alors

$$
t_{H}\left(D_{L^{\prime}}^{\prime}\right) \leq t_{N}\left(D^{\prime}\right)
$$
$D^{\prime}$.

On fixe un sous-objet $D^{\prime}$ de $D$ et on commence par établir certaines propriétés de

Définition 4.12. Soient $E, E^{\prime}$ deux bons sous-objets de $D$ tels que $\operatorname{dim} E^{\prime}>\operatorname{dim} E$, on définit

$$
\alpha\left(E^{\prime} / E, D^{\prime}\right)=\frac{\operatorname{dim} E^{\prime} \cap D^{\prime}-\operatorname{dim} E \cap D^{\prime}}{\operatorname{dim} E^{\prime}-\operatorname{dim} E} .
$$

On note $\alpha\left(E^{\prime} / E\right)=\alpha\left(E^{\prime} / E, D^{\prime}\right)$ s'il n'y a pas de risque de confusion. On écrit $\alpha\left(E^{\prime}\right)$ si $E=0$.

Remarque 4.13. (1) Le nombre $\alpha\left(E^{\prime} / E\right)$ peut être négatif.

(2) Soient $E_{1}, E_{2}, E_{3}$ trois bons sous-objets de $D$ tels que $\operatorname{dim} E_{1}<\operatorname{dim} E_{2}<\operatorname{dim} E_{3}$, alors ou bien $\alpha\left(E_{3} / E_{2}\right)>\alpha\left(E_{3} / E_{1}\right)>\alpha\left(E_{2} / E_{1}\right)$, ou bien $\alpha\left(E_{2} / E_{1}\right)>\alpha\left(E_{3} / E_{1}\right)>$ $\alpha\left(E_{3} / E_{2}\right)$, ou bien $\alpha\left(E_{2} / E_{1}\right)=\alpha\left(E_{3} / E_{1}\right)=\alpha\left(E_{3} / E_{2}\right)$. 
Théorème 4.14. (1) Pour tout sous-objet $D^{\prime}$ of $D$, il existe un bon drapeau

$$
\Delta=\Delta_{D^{\prime}}: 0=E_{0} \subsetneq E_{1} \subsetneq \cdots \subsetneq E_{m+1}=D
$$

tel que

(a) pour $1 \leq i \leq m, \alpha\left(E_{i+1} / E_{i}\right) \leq \alpha\left(E_{i} / E_{i-1}\right)$; si $\alpha\left(E_{i+1} / E_{i}\right)=\alpha\left(E_{i} / E_{i-1}\right)$, alors $\operatorname{dim} E_{i+1} / E_{i} \geq \operatorname{dim} E_{i} / E_{i-1} ;$

(b) $N\left(E_{i}\right) \subset E_{i-1}$;

(c) pour tout $i$, il existe un entier $j \leq 1$, tel que $\left(\varphi^{\prime}-q^{\prime j} a\right) E_{i} \subset E_{i-1}$;

(d) $\Delta$ n'a pas de sous-drapeau qui est bon et qui vérifie les conditions (a)-(c).

(2) $\mathrm{Si}$

$$
\Delta^{\prime}=\Delta_{D^{\prime}}^{\prime}: 0=E_{0}^{\prime} \subsetneq E_{1}^{\prime} \subsetneq \cdots \subsetneq E_{m^{\prime}+1}^{\prime}=D
$$

est un autre bon drapeau vérifiant les propriétés (a)-(d), alors $m=m^{\prime}$ et

$$
\operatorname{dim} E_{i}=\operatorname{dim} E_{i}^{\prime}, \quad \alpha\left(E_{i+1} / E_{i}\right)=\alpha\left(E_{i+1}^{\prime} / E_{i}^{\prime}\right)
$$

pour tout $i$.

Démonstration. (1) On munit $\mathbb{Q} \times \mathbb{N}$ de l'ordre suivant :

$$
\left(x_{1}, y_{1}\right) \geq\left(x_{2}, y_{2}\right) \Leftrightarrow\left(x_{1}>x_{2}\right) \text { ou }\left(x_{1}=x_{2} \text { et } y_{1} \leq y_{2}\right) .
$$

On considère l'ensemble des bons sous-objets non nuls de $D$ et on choisit un d'entre eux, noté $E_{1}$, tel que le couple $\left(\alpha\left(E_{1}\right), \operatorname{dim} E_{1}\right)$ soit maximal. Puis on considère l'ensemble des bons sous-objets de $D$ contenant $E_{1}$ strictement et on choisit un d'entre eux, noté $E_{2}$, tel que $\left(\alpha\left(E_{2} / E_{1}\right), \operatorname{dim} E_{2} / E_{1}\right)$ soit maximal, et on obtient ainsi un bon drapeau

$$
0=E_{0} \subsetneq E_{1} \subsetneq \cdots \subsetneq E_{m} \subsetneq E_{m+1}=D .
$$

On prouve maintenant que ce drapeau vérifie les conditions (a)-(d) :

(a) et (d) sont automatiques et proviennent de la définition du drapeau.

(b) Supposons d'abord $i=1$. Comme

$$
\operatorname{dim} E_{1}=\operatorname{dim} N\left(E_{1}\right)+\operatorname{dim} E_{1}^{N=0}
$$

et

$$
\operatorname{dim}\left(E_{1} \cap D^{\prime}\right) \leq \operatorname{dim} N\left(E_{1}\right) \cap D^{\prime}+\operatorname{dim}\left(E_{1}^{N=0} \cap D^{\prime}\right),
$$

on déduit que ou bien $\alpha\left(N\left(E_{i}\right)\right) \geq \alpha\left(E_{1}\right)$ ou bien $\alpha\left(E_{1}^{N=0}\right) \geq \alpha\left(E_{1}\right)$, et donc, par définition de $E_{1}, N\left(E_{1}\right)=0$ puisque $N\left(E_{1}\right) \neq E_{1}$.

Pour $i$ général, on considère $E_{i+1}^{\prime}=\left\{v \in E_{i+1} \mid N(v) \in E_{i}\right\}$ qui contient $E_{i}$. On a une suite exacte de $L_{0}^{\prime} \otimes_{\mathbb{Q}_{p}} K$-modules libres

$$
0 \rightarrow E_{i+1}^{\prime} / E_{i} \rightarrow E_{i+1} / E_{i} \rightarrow\left(E_{i}+N\left(E_{i+1}\right)\right) / E_{i} \rightarrow 0,
$$

donc d'après le choix de $E_{i}$, on obtient

$$
E_{i}+N\left(E_{i+1}\right)=E_{i} \text { ou } E_{i+1} .
$$

Or $\operatorname{ker}\left(\left.N\right|_{E_{i+1}}\right) \neq 0$, on obtient $N\left(E_{i+1}\right) \subseteq E_{i}$. 
(c) Supposons d'abord $i=1$. Comme

$$
E_{1}=\left(E_{1} \cap D_{=0}\right) \oplus \cdots \oplus\left(E_{1} \cap D_{=s}\right),
$$

et

$$
D^{\prime} \cap E_{1}=\left(D^{\prime} \cap\left(E_{1} \cap D_{=0}\right)\right) \oplus \cdots \oplus\left(D^{\prime} \cap\left(E_{1} \cap D_{=s}\right)\right),
$$

où $D^{\prime} \cap\left(E_{1} \cap D_{=j}\right)$ est le sous-espace de $D^{\prime} \cap E_{1}$ formé par les vecteurs $v$ tels que $\left(\varphi-q^{\prime j} a\right)^{k} v=0$ si $k \gg 0$. Le choix de $E_{1}$ implique $E_{1}=E_{1} \cap D_{=j}$ pour un $j$, i.e., $E_{1} \subset D_{=j}$. En utilisant la suite exacte

$$
0 \rightarrow \operatorname{ker}\left(\left.\left(\varphi^{\prime}-q^{\prime j} a\right)\right|_{E_{1}}\right) \rightarrow E_{1} \rightarrow\left(\varphi^{\prime}-q^{\prime j} a\right)\left(E_{1}\right) \rightarrow 0
$$

on obtient $\left(\varphi^{\prime}-q^{\prime j} a\right)\left(E_{1}\right)=0$. Dans le cas général, on utilise l'argument analogue comme en (b).

(2) Cela sera une conséquence du lemme ci-dessous.

Lemme 4.15. On fixe un drapeau $\Delta_{D^{\prime}}$ comme dans le théorème. Pour tout bon sousobjet $L$ de $D$, soit $i \in \mathbb{Z}_{\geq 0}$ l'unique entier tel que

$$
\operatorname{dim} E_{i}<\operatorname{dim} L \leq \operatorname{dim} E_{i+1} .
$$

Alors on a $\alpha\left(L / E_{i}\right) \leq \alpha\left(E_{i+1} / E_{i}\right)$. En particulier, si $\operatorname{dim} L=\operatorname{dim} E_{i+1}$, alors

$$
\operatorname{dim} D^{\prime} \cap L \leq \operatorname{dim} D^{\prime} \cap E_{i+1} .
$$

Démonstration. Supposons l'énoncé faux et $L$ un tel sous-objet de $D$ de dimension maximale. Soit $i$ l'unique entier tel que $\operatorname{dim} E_{i}<\operatorname{dim} L \leq \operatorname{dim} E_{i+1}$. Alors par hypothèse, $\alpha\left(L / E_{i}\right)>\alpha\left(E_{i+1} / E_{i}\right)$.

Évidemment $L \neq D$. Soit $j$ le plus petit entier tel que $E_{j+1} \nsubseteq L L$, de sorte que $j \leq i$ et $E_{j} \subseteq L \cap E_{j+1}$. On pose $L^{\prime}=L+E_{j+1}$ qui contient $L$ strictement et soit $l$ l'unique entier tel que $\operatorname{dim} E_{l}<\operatorname{dim} L^{\prime} \leq \operatorname{dim} E_{l+1}$ (alors $l \geq i$ ). On voit qu'il suffit de prouver que

$$
\alpha\left(L^{\prime} / E_{l}\right)>\alpha\left(E_{l+1} / E_{l}\right) .
$$

Notons que $L \cap E_{j+1}$ est bon et contient $E_{j}$, on a $\alpha\left(E_{j+1} /\left(L \cap E_{j+1}\right)\right) \geq \alpha\left(E_{j+1} / E_{j}\right)$ par le choix de $E_{j+1}$, et donc

$$
\alpha\left(E_{j+1} /\left(L \cap E_{j+1}\right)\right) \geq \alpha\left(E_{i+1} / E_{i}\right) .
$$

D'autre part, on a évidemment $\alpha\left(L^{\prime} / L\right) \geq \alpha\left(E_{j+1} /\left(L \cap E_{j+1}\right)\right)$. Donc on obtient enfin $\alpha\left(L^{\prime} / E_{i}\right)>\alpha\left(E_{i+1} / E_{i}\right)$, et ceci achève la preuve dans le cas $l=i$. On est donc ramené au cas $l>i$. Dans ce cas on a $\alpha\left(L^{\prime} / E_{i}\right)>\alpha\left(E_{i+1} / E_{i}\right) \geq \alpha\left(E_{l} / E_{i}\right)$, et ceci implique $\alpha\left(L^{\prime} / E_{l}\right)>\alpha\left(E_{l} / E_{i}\right) \geq \alpha\left(E_{l+1} / E_{l}\right)$.

Remarque 4.16. D'après (b) et (c) du théorème 4.14, on a pour tout $i$, qu'il existe $j$, $l$ et une injection $E_{i+1} / E_{i} \rightarrow D_{=j, l+1} / D_{=j, l}$. 
On fixe un bon drapeau de $D$

$$
\Delta=\Delta_{D^{\prime}}: 0=E_{0} \subsetneq E_{1} \subsetneq \cdots \subsetneq E_{m+1}=D
$$

comme dans le théorème 4.14.

Soient $F_{1}$ le plus grand bon sous-objet tel que $\alpha\left(F_{1}\right)=1$ et $F_{2}$ le plus grand bon sous-objet contenant $D^{\prime}$. D'après le lemme 4.15, on voit que $F_{1}$ et $F_{2}$ appartiennent à $\Delta$. On note $k^{\prime}$ et $k$ les entiers tels que $F_{1}=E_{k^{\prime}}$ et $F_{2}=E_{k^{\prime}+k}$ (indépendants du choix du drapeau). On considère le drapeau

$$
0=E_{0} \subsetneq E_{k^{\prime}}=F_{1} \subsetneq \cdots \subsetneq E_{k^{\prime}+k}=F_{2} \subsetneq E_{m+1}=D,
$$

et on pose $a_{0}=\operatorname{dim} F_{1}, a_{k+1}=\operatorname{dim} D-\operatorname{dim} F_{2}$, et pour $1 \leq i \leq k$

$$
a_{i}=\operatorname{dim} E_{k^{\prime}+i} / E_{k^{\prime}+i-1}, \quad c_{i}=\operatorname{dim} D^{\prime} \cap E_{k^{\prime}+i} / D^{\prime} \cap E_{k^{\prime}+i-1} .
$$

Proposition 4.17. Avec les notations plus haut, on a

$$
a_{0} \geq \max \left\{c_{i}\right\}_{1 \leq i \leq k}, \quad a_{k+1} \geq \max \left\{a_{i}-c_{i}\right\}_{1 \leq i \leq k} .
$$

Démonstration. On prouve seulement la première inégalité, la preuve de l'autre étant analogue. D'aprè s l'assertion (i) du lemme 4.18 ci-dessous, on peut supposer $h=$ $\operatorname{dim} D_{0}=1$.

Choisissons un $r$ tel que $c_{r}=\max _{1 \leq j \leq k}\left\{c_{j}\right\}$. On sait qu'il existe $j, l$ tels que $E_{k^{\prime}+r} / E_{k^{\prime}+r-1} \hookrightarrow D_{=j, l+1} / D_{=j, l}$. On a donc $\operatorname{dim} D^{\prime} \cap D_{=j, l+1}-\operatorname{dim} D^{\prime} \cap D_{=j, l} \geq c_{r}$, et puis $\operatorname{dim} D^{\prime} \cap D_{=j, 1} \geq c_{r}$ parce que l'on a un homomorphisme injectif

$$
\left(\varphi^{\prime}-q^{\prime j} a\right): D_{=j, l+1} / D_{=j, l} \rightarrow D_{=j, l} / D_{=j, l-1} .
$$

D'après le lemme 4.18 ci-dessous, on a pour tout $1 \leq i \leq j$,

$$
\operatorname{dim} D^{\prime} \cap D_{=i-1,1} \geq \operatorname{dim} D^{\prime} \cap D_{=i, 1}-1,
$$

et pour que $\operatorname{dim} D^{\prime} \cap D_{=i-1,1}=\operatorname{dim} D^{\prime} \cap D_{=i, 1}-1$, il faut $\operatorname{dim} D_{=i-1,1}=\operatorname{dim} D_{=i, 1}-1$, d'où

$$
\operatorname{dim} \operatorname{ker}\left(\left.N\right|_{D_{=i, 1}}\right)=1,\left.\quad \operatorname{ker} N\right|_{D_{=i, 1}} \subset D^{\prime} .
$$

S'il en est ainsi, alors par la définition de $F_{1}$ on a

$$
\operatorname{ker}\left(\left.N\right|_{D_{=i, 1}}\right) \subset F_{1},
$$

et ceci permet de conclure puisque $\operatorname{dim} D^{\prime} \cap D_{=1,1} \leq 1$ d'après le lemme 4.18 .

Lemme 4.18. (1) Pour tout sous-objet $\bar{D}$ de $D$, on a $h \mid \operatorname{dim} \bar{D}$.

(2) Pour tout $k, \operatorname{dim} \operatorname{ker} N \cap \operatorname{ker}\left(\varphi^{\prime}-q^{\prime k} a\right)=h$ ou 0 .

(3) $\operatorname{dim} D_{=1,1}=h,\left|\operatorname{dim} D_{=i, 1}-\operatorname{dim} D_{=i-1,1}\right|=h$ ou 0 .

Démonstration. (1) Comme $\bar{D}=\bigoplus_{j=1}^{s} \bar{D} \cap D_{=j}$, on peut supposer $N=0$ et alors par définition, $\left(\varphi, \operatorname{Gal}\left(L^{\prime} / L\right), D\right)$ est une extension successive de objets de type $\left(\left.\varphi\right|_{D_{0}}, \operatorname{Gal}\left(L^{\prime} / L\right)\right.$, $\left.D_{0}\right)$ qui est absolument irréductible. L'énoncé s'en déduit.

(2) Cela résulte de la définition de $\varphi$ et de (1).

(3) Le premier énoncé est une conséquence directe de (2). Pour le deuxième, on considère $N\left(D_{=i, 1}\right)$. D'une part, on a $\operatorname{dim} D_{=i, 1}-\operatorname{dim} N\left(D_{=i, 1}\right) \leq h$ d'après (2). D'autre part, $\operatorname{dim} D_{=i-1,1}-\operatorname{dim} N\left(D_{=i, 1}\right) \leq h$ d'après la définition de $\varphi$. Enfin, l'assertion (i) nous permet de conclure. 
En vue du théorème 4.14 et de la proposition 4.17, on a besoin de la définition suivante :

Définition 4.19. Soient $\alpha=\left(a_{0}, \ldots, a_{k+1}\right)$ une partition de $d+1$ et $\Omega$ un sous-ensemble de $\{1, \ldots, d+1\}$. Posons

$I_{0}=\left\{1,2, \ldots, a_{0}\right\}, I_{1}=\left\{a_{0}+1, \ldots, a_{0}+a_{1}\right\}, \cdots, I_{k+1}=\left\{a_{0}+\cdots+a_{k}+1, \ldots, d+1\right\}$,

et $c_{i}=\left|\Omega \cap I_{i}\right|$ pour $0 \leq i \leq k+1$. On dit que la paire $(\Omega, \alpha)$ est de type spécial si les nombres $\left\{a_{i}\right\}_{0 \leq i \leq k+1}$ et $\left\{c_{i}\right\}_{0 \leq i \leq k+1}$ vérifient les conditions suivantes :

(i) $a_{0}=c_{0}>0, c_{k+1}=0$, et $a_{i} \geq c_{i}>0$ pour $1 \leq i \leq k$,

(ii) $\frac{c_{i}}{a_{i}} \geq \frac{c_{i+1}}{a_{i+1}}$ pour tout $i$,

(iii) $a_{0} \geq \max _{1 \leq i \leq k}\left\{c_{i}\right\}, a_{k+1} \geq \max _{1 \leq i \leq k}\left\{a_{i}-c_{i}\right\}$.

Les paires de types spéciaux ont la propriété suivante :

Proposition 4.20. Soient $(\Omega, \alpha)$ une paire de type spécial, et $\left\{m_{i}\right\}_{1 \leq i \leq d+1},\left\{n_{i}\right\}_{1 \leq i \leq d+1}$ des nombres réels tels que

(a) $m_{i} \leq m_{i+1}, n_{i} \leq n_{i+1}$

(b) $m_{i+1}-m_{i} \geq n_{i+1}-n_{i}$,

(c) $\sum_{i=1}^{d+1} m_{i} \leq \sum_{i=1}^{d+1} n_{i}$

Alors on $a \sum_{i \in \Omega} m_{i} \leq \sum_{i \in \Omega} n_{i}$.

Remarque 4.21. Cette proposition peut être vue comme une généralisation de [3], lemme 5.4.

La démonstration du théorème 4.11 utilise la proposition 4.20, dont la preuve sera donnée au paragraphe suivant.

Démonstration de 4.11. Reprenons les notations de la proposition 4.17. Donc on a $\sum_{i=0}^{k+1} a_{i}=$ $d+1$. Notons $\alpha=\left(a_{0}, \ldots, a_{k+1}\right)$ la partition de $d+1$,

$$
I_{0}=\left\{1, \ldots, a_{0}\right\}, \cdots, I_{k+1}=\left\{a_{0}+\cdots+a_{k}+1, \ldots, d+1\right\},
$$

et $\Omega$ le sous-ensemble de $\{1, \ldots, d+1\}$ tel que

$$
\Omega \cap I_{i}=\left\{\sum_{j=0}^{i} a_{j}-c_{i}+1, \sum_{j=0}^{i} a_{j}-c_{i}+2, \ldots, \sum_{j=0}^{i} a_{j}\right\} .
$$

On sait que, d'après le théorème 4.14 et la proposition 4.17, $(\Omega, \alpha)$ est de type spécial.

On pose $s_{i}=\operatorname{dim}\left(D^{\prime} \cap D_{=i}\right), r_{i}=\sharp\left\{j \in \Omega \mid \operatorname{dim} D_{<i}+1 \leq j \leq \operatorname{dim} D_{\leq i}\right\}$ et

$$
n_{j}= \begin{cases}t_{N}\left(D_{0}\right) & 1 \leq j \leq \operatorname{dim} D_{=0} \\ t_{N}\left(D_{0}(1)\right) & \operatorname{dim} D_{=0}+1 \leq j \leq \operatorname{dim} D_{\leq 1} \\ \vdots & \vdots \\ t_{N}\left(D_{0}(n)\right) & \operatorname{dim} D_{<n}+1 \leq j \leq \operatorname{dim} D_{\leq n}=d+1\end{cases}
$$


de sorte qu'on a (notons que $\left.t_{N}\left(D_{0}(i)\right)=t_{N}\left(D_{0}\right)+i\left[K: \mathbb{Q}_{p}\right]\right)$

$$
t_{N}\left(D^{\prime}\right)=\sum_{i=0}^{n} s_{i} n_{i}=\operatorname{dim} D^{\prime} \cdot t_{N}\left(D_{0}\right)+\left[K: \mathbb{Q}_{p}\right] \sum_{i=0}^{n} i s_{i}
$$

et

$$
\sum_{j \in \Omega} n_{j}=\sum_{i=0}^{n} r_{i} n_{i}=\operatorname{dim} D^{\prime} \cdot t_{N}\left(D_{0}\right)+\left[K: \mathbb{Q}_{p}\right] \sum_{i=0}^{n} i r_{i}
$$

(a) D'abord, on prouve le théorème sous l'hypothèse $\sum_{i=0}^{l} s_{i} \leq \sum_{i=0}^{l} r_{i}$ pour tout $l$. Alors on a :

- $t_{H}\left(D_{L^{\prime}}^{\prime}\right) \leq[K: L] \sum_{j \in \Omega} \sum_{\sigma} i_{j, \sigma}$. Ceci est une conséquence du corollaire 4.10,

- $[K: L] \sum_{j \in \Omega} \sum_{\sigma} i_{j, \sigma} \leq \sum_{j \in \Omega} n_{j}$. En posant $m_{j}=[K: L] \sum_{\sigma} i_{j, \sigma}$, on voit que

$$
m_{j+1}-m_{j} \geq\left[K: \mathbb{Q}_{p}\right] \geq n_{j+1}-n_{j}
$$

puisque que $\operatorname{dim} D_{=i} \neq 0$ pour tout $0 \leq i \leq n$. On déduit l'énoncé de la proposition 4.20 .

$-t_{N}\left(D^{\prime}\right) \geq \sum_{j \in \Omega} n_{j}$. Il suffit de prouver que $\sum_{i=0}^{n} i\left(s_{i}-r_{i}\right) \geq 0$. Mais

$$
\begin{aligned}
\sum_{i=0}^{n} i\left(s_{i}-r_{i}\right) & =n \sum_{i=0}^{n}\left(s_{i}-r_{i}\right)-\sum_{i=0}^{n}(n-i)\left(s_{i}-r_{i}\right) \\
& =n \sum_{i=0}^{n}\left(s_{i}-r_{i}\right)-\sum_{l=0}^{n-1} \sum_{i=0}^{l}\left(s_{i}-r_{i}\right),
\end{aligned}
$$

l'inégalité résulte du fait que $\sum_{i=0}^{n} s_{i}=\sum_{i=0}^{n} r_{i}$ et $\sum_{i=0}^{l}\left(s_{i}-r_{i}\right) \leq 0$ par hypothèse.

Donc on obtient $t_{H}\left(D_{L^{\prime}}^{\prime}\right) \leq t_{N}\left(D^{\prime}\right)$.

(b) Dans le cas général, on voit facilement qu'il suffit de trouver un autre sousensemble $\Omega^{\prime}$ de $\{1, \ldots, d+1\}$ tel que

$-t_{H}\left(D_{L^{\prime}}^{\prime}\right) \leq[K: L] \sum_{j \in \Omega^{\prime}} \sum_{\sigma} i_{j, \sigma}$

- $\left(\Omega^{\prime}, \alpha\right)$ est de type spécial et $\left|\Omega^{\prime} \cap I_{i}\right|=\left|\Omega \cap I_{i}\right|=c_{i}$;

- pour tout $l$

$$
\operatorname{dim}\left(D^{\prime} \cap D_{\leq l}\right) \leq \sharp\left\{j \in \Omega^{\prime} \mid j \leq \operatorname{dim} D_{\leq l}\right\} .
$$

Si $0 \leq m \leq n$, on notera $i(m)$ l'unique entier tel que $0<i(m) \leq k$ et $\operatorname{dim} E_{i(m)-1}<$ $\operatorname{dim} D_{\leq m} \leq \operatorname{dim} E_{i(m)}$. D'abord, soit $m_{1}$ le plus petit entier tel que

$$
\operatorname{dim}\left(D^{\prime} \cap D_{\leq m_{1}}\right)>\sharp\left\{j \in \Omega \mid j \leq \operatorname{dim} D_{\leq m_{1}}\right\} .
$$

Alors si on pose

$$
x_{m_{1}}=\operatorname{dim} D^{\prime} \cap D_{\leq m_{1}}-\operatorname{dim} D^{\prime} \cap E_{i\left(m_{1}\right)-1}
$$

et

$$
y_{m_{1}}=\left(\operatorname{dim} E_{i\left(m_{1}\right)}-\operatorname{dim} D_{\leq m_{1}}\right)-\left(\operatorname{dim} D^{\prime} \cap E_{i\left(m_{1}\right)}-\operatorname{dim} D^{\prime} \cap D_{\leq m_{1}}\right),
$$


on en déduit que $x_{m}>0$ et $y_{m}>0$. On définit un sous-ensemble $\Omega_{1}^{\prime}$ de $\{1, \ldots, d+1\}$, en modifiant la définition de $\Omega$, tel que $\Omega_{1}^{\prime} \cap I_{j}=\Omega \cap I_{j}$ pour $j \neq i\left(m_{1}\right)$ et

$$
\begin{aligned}
\Omega_{1}^{\prime} \cap I_{i\left(m_{1}\right)}= & \left\{\operatorname{dim} D_{\leq m_{1}}-x_{m_{1}}+1, \ldots, \operatorname{dim} D_{\leq m_{1}}\right\} \sqcup \\
& \left\{\operatorname{dim} E_{i\left(m_{1}\right)}-\left(c_{i\left(m_{1}\right)}-x_{m_{1}}\right)+1, \ldots, \operatorname{dim} E_{i\left(m_{1}\right)}\right\} .
\end{aligned}
$$

Notons que $c_{i\left(m_{1}\right)}-x_{m_{1}}>0$ d'après l'hypothèse sur $m_{1}$ et le théorème 4.14, (2). Après, si $\Omega_{1}^{\prime}$ vérifie $(* *)$, on pose $\Omega^{\prime}=\Omega_{1}^{\prime}$; sinon, soit $m_{2}$ le plus petit entier tel que (**) soit faux pour $\Omega_{1}^{\prime}$, alors $m_{2}>m_{1}$ et $y_{m_{2}}>0$ où

$$
y_{m_{2}}=\left(\operatorname{dim} E_{i\left(m_{2}\right)}-\operatorname{dim} D_{\leq m_{2}}\right)-\left(\operatorname{dim} D^{\prime} \cap E_{i\left(m_{2}\right)}-\operatorname{dim} D^{\prime} \cap D_{\leq m_{2}}\right) .
$$

Il y a deux possibilités :

- si $i\left(m_{2}\right)>i\left(m_{1}\right)$, on pose

$$
x_{m_{2}}=\operatorname{dim} D^{\prime} \cap D_{\leq m_{2}}-\operatorname{dim} D^{\prime} \cap E_{i\left(m_{2}\right)-1},
$$

l'hypothèse sur $m_{2}$ implique que $x_{m_{2}}>0$; on définit $\Omega_{2}^{\prime}$ comme précédemment;

- si $i\left(m_{1}\right)=i\left(m_{2}\right)$, on pose

$$
x_{m_{2}}=\operatorname{dim} D^{\prime} \cap D_{\leq m_{2}}-\operatorname{dim} D^{\prime} \cap D_{\leq m_{1}},
$$

de sort que $x_{m_{2}}>0$; on définit $\Omega_{2}^{\prime}$ en modifiant la définition de $\Omega_{1}^{\prime}$ par

$$
\begin{aligned}
& \Omega_{2}^{\prime} \cap\left\{\operatorname{dim} D_{\leq m_{1}}+1, \ldots, \operatorname{dim} E_{i\left(m_{1}\right)}\right\} \\
= & \left\{\operatorname{dim} D_{\leq m_{2}}-x_{m_{2}}+1, \ldots, \operatorname{dim} D_{\leq m_{2}}\right\} \sqcup \\
& \left\{\operatorname{dim} E_{i\left(m_{1}\right)}-\left(c_{i\left(m_{1}\right)}-x_{m_{1}}-x_{m_{2}}\right)+1, \ldots, \operatorname{dim} E_{i\left(m_{1}\right)}\right\} .
\end{aligned}
$$

Par récurrence on obtient bien un sous-ensemble $\Omega^{\prime}$ de $\{1, \ldots, d+1\}$ vérifiant les conditions demandées : les deux dernières résultent de la définition et la première résulte encore du corollaire 4.10 puisque $D_{\leq i}$ est aussi un bon sous-objet de $D$.

\subsection{Preuve de 4.20}

Dans ce paragraphe, on prouve la proposition 4.20, On commence par un lemme :

Lemme 4.22. Soient $k \geq 1$ et $\left\{a_{i}\right\}_{0 \leq i \leq k+1},\left\{c_{i}\right\}_{0 \leq i \leq k+1}$ des nombres réels vérifiant les conditions (i)-(iii) de la définition 4.19, alors il existe des nombres réels $\left\{t_{i}\right\}_{1 \leq i \leq k}$ tels que

(i) ' $\frac{t_{1}}{a_{0}}=\frac{t_{2}}{c_{1}}=\cdots=\frac{t_{k}}{c_{k-1}}=: r$,

(ii)' pour tout $1 \leq l \leq k$,

$$
\sum_{i=1}^{l} t_{i} \geq \sum_{i=1}^{l}\left(a_{i}-c_{i}\right)
$$

(iii) $\sum_{i=1}^{k} t_{i}-\sum_{i=1}^{k}\left(a_{i}-c_{i}\right)+r c_{k} \leq a_{k+1}$. 
Démonstration. On peut écrire, si les $t_{i}$ existent, $t_{i}=t_{1} c_{i-1} / a_{0}$ pour $1 \leq i \leq k$, et alors (ii)' se réduit à

$$
t_{1}\left(1+\frac{1}{a_{0}} \sum_{i=1}^{l-1} c_{i}\right) \geq \sum_{i=1}^{l}\left(a_{i}-c_{i}\right)
$$

et (iii)' se réduit à

$$
t_{1}\left(1+\frac{1}{a_{0}} \sum_{i=1}^{k} c_{i}\right) \leq a_{k+1}+\sum_{i=1}^{k}\left(a_{i}-c_{i}\right)
$$

On prend $t_{1}=\max _{1 \leq l \leq k}\left\{\left(\sum_{i=1}^{l}\left(a_{i}-c_{i}\right)\right)\left(1+\frac{1}{a_{0}} \sum_{i=1}^{l-1} c_{i}\right)^{-1}\right\}$, et $t_{i}=t_{1} c_{i-1} / a_{0}$, alors (i)', (ii)' sont satisfaits. Pour voir que les $t_{i}$ ainsi définis satisfont à (iii)', il suffit de vérifier que pour $1 \leq l \leq k$,

$$
\left(\sum_{i=1}^{l}\left(a_{i}-c_{i}\right)\right)\left(1+\frac{1}{a_{0}} \sum_{i=1}^{k} c_{i}\right) \leq\left(1+\frac{1}{a_{0}} \sum_{i=1}^{l-1} c_{i}\right)\left(a_{k+1}+\sum_{i=1}^{k}\left(a_{i}-c_{i}\right)\right),
$$

qui est une conséquence facile du (ii) et (iii).

Dans la suite de cet article, si on se donne les $\left\{a_{i}\right\}_{0 \leq i \leq k+1}$ et $\left\{c_{i}\right\}_{0 \leq i \leq k+1}$ comme dans le lemme 4.22, on prendra les $\left\{t_{i}\right\}_{1 \leq i \leq k}$ et $r$ comme dans le lemme tel que $r$ est le plus petit possible.

Démonstration de 4.20. Grâce à la condition (b) $: m_{i+1}-m_{i} \geq n_{i+1}-n_{i}$, on voit qu'on peut supposer

$$
\Omega=\left\{1 \leq j \leq d+1 \mid \text { il existe } l \text { tel que } \sum_{i=0}^{l} a_{i}-c_{l}+1 \leq j \leq \sum_{i=0}^{l} a_{i}\right\} .
$$

Posons $I=[0, d+1[$. On prend deux fonctions lisses $f, g: I \rightarrow \mathbb{R}$ telles que (c'est toujours possible)

- $f^{\prime}(x) \geq g^{\prime}(x) \geq 0$, et $\int_{I} f \leq \int_{I} g$, et

- pour $0 \leq i \leq d, f(i)=m_{i+1}, g(i)=n_{i+1}$.

Le lemme ci-dessus nous permet de définir des nombres $\left(t_{i}\right)_{1 \leq i \leq k}$ vérifiant les conditions (i)'-(iii)'. Posons

$$
J_{i}= \begin{cases}{\left[0, a_{0}[\right.} & \text { si } i=0 \\ {\left[\sum_{j=0}^{i} a_{j}-c_{i}, \sum_{j=0}^{i} a_{j}[\right.} & \text { si } 1 \leq i \leq k \\ \emptyset & \text { si } i=k+1\end{cases}
$$

et

$$
J_{i}^{\prime}= \begin{cases}{\left[0, a_{0}[\right.} & \text { si } i=0 \\ {\left[a_{0}+\sum_{j=1}^{i-1}\left(t_{j}+c_{j}\right)+t_{i}, a_{0}+\sum_{j=0}^{i}\left(a_{j}+c_{j}\right)[\right.} & \text { si } 1 \leq i \leq k \\ \emptyset & \text { si } i=k+1\end{cases}
$$


Alors on a $\left|J_{i}\right|=\left|J_{i}^{\prime}\right|, \bigsqcup_{1 \leq i \leq k} J_{i}^{\prime} \subset I$ et

$$
\sum_{i \in \Omega}\left(m_{i}-n_{i}\right) \leq \sum_{i=0}^{k} \int_{J_{i}}(f-g) \leq \sum_{i=0}^{k} \int_{J_{i}^{\prime}}(f-g) \leq \frac{1}{r+1} \int_{I}(f-g) \leq 0,
$$

ce qui permet de conclure.

\subsection{Le cas général}

On considère le cas général : $D=\bigoplus_{i=1}^{v} D_{H_{i}}$. D'abord, la proposition 4.20 se généralise aisément :

Proposition 4.23. Soit $\alpha=\left(d_{1}, \ldots, d_{v}\right)$ une partition de $d+1$, et pour tout $1 \leq i \leq v$ soient $\alpha_{i}$ une partition de $d_{i}$ et $\Omega_{i}$ un sous-ensemble de $\left\{1, \ldots, d_{i}\right\}$ tels que la paire $\left(\Omega_{i}, \alpha_{i}\right)$ est de type spécial. On associe, à chaque paire $\left(\Omega_{i}, \alpha_{i}\right)$, le nombre réel positif $r_{i}$ comme dans le lemme 4.22, et on suppose $r_{1} \geq r_{2} \geq \cdots \geq r_{v}$. Posons

$$
\Omega_{i}^{\prime}=\left\{d_{1}+\cdots d_{i-1}+l \mid l \in \Omega_{i}\right\}, \quad \Omega=\bigsqcup_{i} \Omega_{i}^{\prime}
$$

de sorte que $\Omega$ est un sous-ensemble de $\{1, \ldots, d+1\}$. De plus, soient $\left\{m_{i}\right\}_{1 \leq i \leq d+1}$ et $\left\{n_{i}\right\}_{1 \leq i \leq k+1}$ des nombres réels vérifiant les conditions (a)-(c) de la proposition 4.20, alors on $a \sum_{j \in \Omega} m_{j} \leq \sum_{j \in \Omega} n_{j}$.

La preuve de cette proposition est analogue à celle de 4.20 et on laisse les détails au lecteur.

Maintenant, posons $d_{i}=\operatorname{dim} D_{H_{i}}$, et $D_{i}^{\prime}=D^{\prime} \cap D_{H_{i}}$ pour tout $1 \leq i \leq v$ et tout sous-objet $D^{\prime}$ de $D$. Comme $D_{i}^{\prime}$ est un sous-objet de $D_{H_{i}}$, on peut lui associer un drapeau $\Delta_{i}=\Delta_{D_{i}^{\prime}}$ d'après le théorème 4.14, et puis un sous-ensemble $\Omega_{i}$ de $\left\{1, \ldots, d_{i}\right\}$ et un nombre réel positif $r_{i}$ comme dans le lemme 4.22. On peut supposer $r_{1} \geq r_{2} \geq \cdots \geq r_{v}$. Posons

$$
\Omega_{i}^{\prime}=\left\{d_{1}+\cdots d_{i-1}+l \mid l \in \Omega_{i}\right\}, \quad \text { et } \Omega=\bigsqcup_{i} \Omega_{i}^{\prime} .
$$

On a alors $t_{H}\left(D^{\prime}\right) \leq \sum_{j \in \Omega} \sum_{\sigma} i_{j, \sigma}$ par le corollaire 4.10. Un argument analogue à la preuve du corollaire 4.11, en utilisant la proposition ci-dessus, nous permet de conclure.

\section{Références}

[1] L. Berger, C. Breuil, Sur quelques représentations potentiellement cristallines de $\mathrm{GL}_{2}\left(\mathbb{Q}_{p}\right)$, prépublication I.H.E.S, 2006.

[2] C. Breuil, A. Mézard, Multiplicités modulaires et représentations de $\mathrm{GL}_{2}\left(\mathbb{Z}_{p}\right)$ et de $\operatorname{Gal}\left(\overline{\mathbb{Q}}_{p} / \mathbb{Q}_{p}\right)$ en $l=p$, Duke Math. J. 115 (2002), 205-310.

[3] C. Breuil \& P. Schneider, First steps towards p-adic Langlands functoriality, à paraître dans J. Reine Angew. Math.

[4] W. Casselman, Introduction to the theory of admissible representations of p-adic reductive groups, preprint, 1995. 
[5] L. Clozel, Motifs et formes automorphes : applications du principe de functorialité, Perspectives in Math. 10, Academic Press, 1990, 77-159.

[6] P. Colmez, Une correspondance de Langlands locale p-adique pour les représentations semi-stables de dimension 2, prépublication, 2006.

[7] P. Deligne, Les constantes des équations fonctionnelles des fonctions L, Lecture Notes in Math. 349, Springer-Verlag, 1973, 501-597.

[8] M. Emerton, Locally analytic vectors in representations of locally p-adic analytic groups, à paraître dans Memoirs of the AMS.

[9] M. Emerton, p-adic L-functions and completions of representations of p-adic reductive groups, Duke Math. J. 130 (2005), no. 2, 353-392.

[10] M. Emerton, Jacquet modules of locally analytic representations of $p$-adic reductive groups I. Construction and first properties Appeared in Ann. Sci. E.N.S. 39 (2006), no. 5, 775-839.

[11] J.M. Fontaine, Représentations p-adiques semi-stables, Astérisque 223, Soc. Math. de France, 1994, 113-184.

[12] J.M. Fontaine, Représentations l-adiques potentiellement semi-stables, Astérisque 223, Soc. Math. de France, 1994, 321-347.

[13] J.M. Fontaine \& M. Rapoport, Existence de filtrations admissibles sur des isocristaux, Bull. Soc. Math. de France 133 (2005), no. 1, 73-86.

[14] S. Kudla, The local Langlands correspondence : the non-Archimedean case, Proc. Symp. Pure Math. 55, Amer. Math. Soc., 1994, 365-410.

[15] P. Schneider \& J. Teitelbaum Banach-Hecke algebras and p-adic Galois representations, Documenta Mathematica, The Book Series 4 (J. Coates' Sixtieth Birthday), 2006, 631-684.

[16] M.-F. Vigneras, Représentations l-modulaires d'un groupe réductif p-adique avec $l \neq p$, Prog. Math., vol. 137, Birkhäuser, 1996.

[17] A. Zelevinsky, Induced representations of reductive p-adic groups II, Ann. Sci. E. N. S. 13 (1980), 165-210.

Département de Mathématiques, bâtiment 425, Université de Paris-Sud, 91405 Orsay cedex, France

E-mail : yongquan.hu@math.u-psud.fr 